\title{
Towards the survey of Entiminae weevils (Coleoptera: Curculionidae) of Russia: species occurring in the Volga and Ural Regions
}

\section{К инвентаризаџии фауны долгоносиков подсемейства Entiminae (Coleoptera: Curculionidae) России: виды, распространенные в Поволжье и на Урале}

\author{
N.N. Yunakov ${ }^{1,2}$, S.V. Dedyukhin ${ }^{3}$ \& R.V. Filimonov ${ }^{4}$ \\ Н.Н. Юнаков ${ }^{1,2}$, С.В. Аедюхин ${ }^{3}$, Р.В. Филимонов ${ }^{4}$
}

\footnotetext{
${ }^{1}$ Zoologisk Museum, University of Oslo, P.O. Box 1172 Blindern, NO-0318, Oslo, Norway; e-mail: n.yunakov@gmail.com

2 Зоологический институт РАН, Университетская наб. 1, Санкт-Петербург 199034, Россия.

${ }^{2}$ Zoological Institute, Russian Academy of Sciences, Universitetskaya nab. 1, St. Petersburg 199034, Russia.

3 Удмуртский государственный университет, Университетская ул. 1, Ижевск 426034, Россия.

${ }^{3}$ Udmurt State University, Universitetskaya str. 1, Izhevsk 426034, Russia; e-mail: ded@uni.udm.ru

4 Русское энтомологическое общество, Университетская наб. 1, Санкт-Петербург 199034, Россия.

${ }^{4}$ Russian Entomological Society, Universitetskaya nab. 1, St. Petersburg 199034, Russia; e-mail: RostVF@yandex.ru
}

KEY WORDS: Entiminae, Curculionidae, fauna of Russia, Volga region, Ural region, checklist. КЛЮЧЕВЫЕ СЛОВА: Entiminae, Curculionidae, фауна России, Поволжье, Урал, список.

ABSTRACT: 116 species and 43 genera of Entiminae weevils are recorded in Volga and Ural regions. Checklist comprises up to date data on the distribution of Entiminae weevils of Eastern part of European Russia. Phacephorus hirtellus Faust, 1885 and Polydrusus obliquatus Faust, 1884 are first records in Russia and Europe. Trichalophus korotyaevi Zherikhin et Nazarov, 1990 is a first record of the genus Trichalophus LeConte, 1876 in recent fauna of Europe.

РЕЗЮМЕ: В Поволжье и на Урале обнаружены 116 видов из 43 родов Entiminae. Список включает детальные сведения о распространении долгоносиков подсемейства Entiminae Востока Европейской части России. Phacephorus hirtellus Faust, 1885 и Polydrusus obliquatus Faust, 1884 впервые обнаружены в России. Trichalophus korotyaevi Zherikhin et Nazarov, 1990 - первая находка рода Trichalophus LeConte, 1876 в современной фауне Европы.

\section{Introduction}

Distribution of Entiminae within certain Russian provinces is still unclear. Old literature data are frequently limited by scarce records like "Eastern European Russia" or "Ural". A number of data are spread among poorly known editions, some other records based on misidentified specimens. Recent workers studying Curculionoidea cover only few provinces by those attentions. Several provinces among European Russia are still out of knowledge of weevils. Well studied provinces Ulyanovsk, Kirov, Udmurtia,
Chuvashia, and Mordovia neighbor with poorly studied Mari El, Tatarstan, and Samara; despite all that the references dealing with Ural fauna are in big abundance, they slightly concern Entiminae (Table 1). Additionally some details on distribution of Entiminae in Volga and Ural Regions are fragmented in the taxonomic revisions [Reitter, 1915; Davidian, 1992; Ismailova, 1993; Arzanov \& Davidian, 1995; Yunakov, 2005; Yunakov \& Korotyaev, 2007]. For easy access to entimins of Volga and Ural the regional keys to weevils may be served [Arnoldi et al., 1965; Isaev, 2007].

Proposed work deals with survey of weevil subfamily Entiminae of Russia, and starts with report on species occurring in Volga and Ural Regions and up to date published records. Before completing this paper, we have established following main goals: (1) to develop convenient universal coding system of macroregions and provinces of Russia which will be subsequently employed for representation of distribution data on weevils of entire Russian fauna; (2) to examine all available Entiminae material from Volga and Ural regions, as well as up to date references; (3) to check the doubtful records.

Following the biogeorgaphic eligibility, we conserve a continuum of fauna presented in the list. As result, some provinces were omitted and the boundaries of this survey became outlined by Ural Mountains: Bashkortostan, Chelyabinsk, Komi, Yekaterinburg (incl. Sverdlovsk Prov.), Orenburg, Perm'; and by Middle course of Volga river basin: Chuvashia, Kirov, Mari El, Mordovia, Samara, Tatarstan, Udmurtia, Ulyanovsk. (Fig. 1, Table 1). 


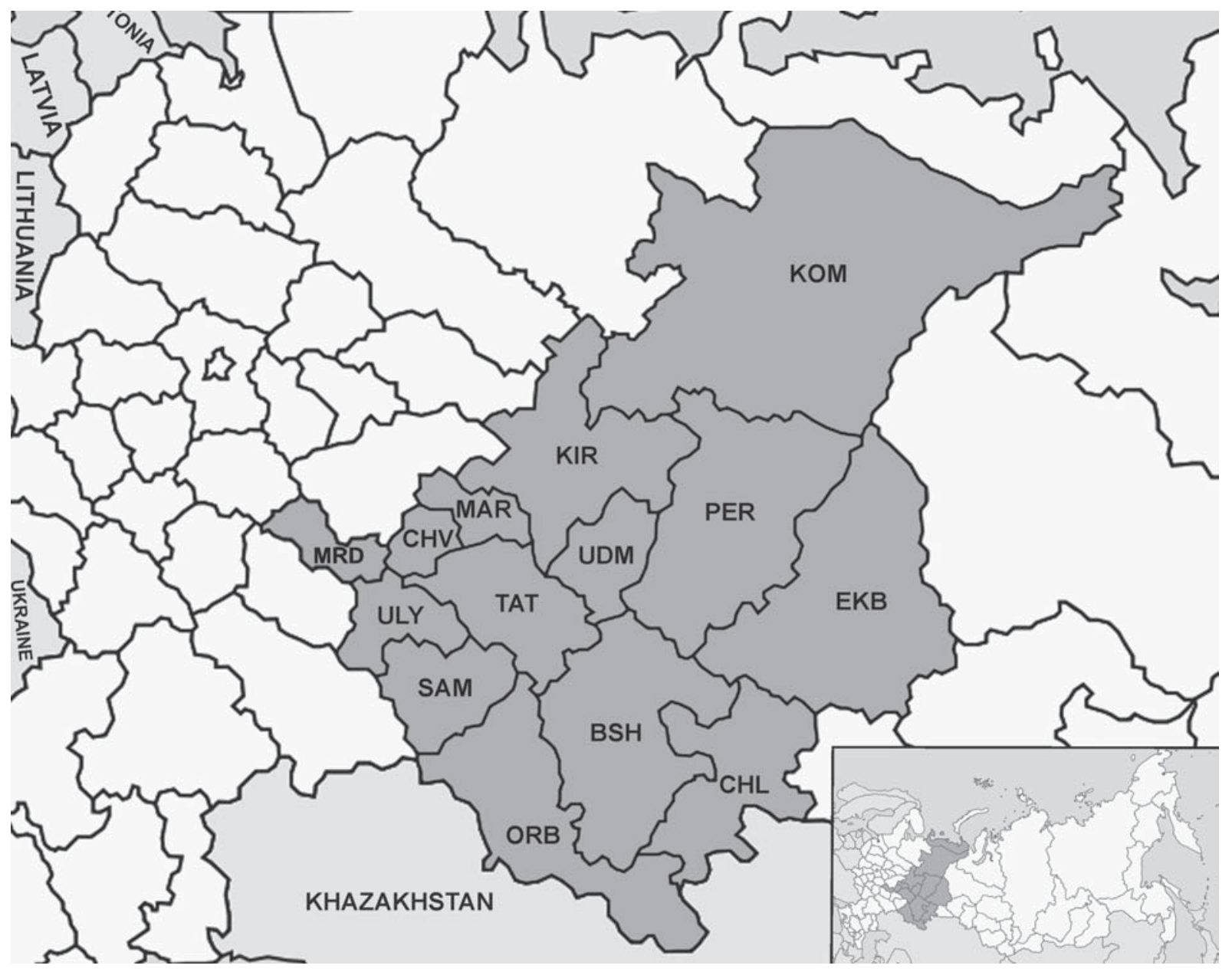

Fig. 1. Map of Volga and Ural Regions and their subdivisions. Thi explanation as in Table 1.

Рис. 1. Карта Поволжья и Урала и их подразделений. Обозначения см. в Таблице 1.

\section{Material and methods}

The checklist basically follows the systematics in order of recent catalogue of weevils [Alonso-Zarazaga \& Lyal, 1999]. Synonymy follows commonly acknowledged synonyms reported from Volga-Ural region. All misidentifications included in the list of synonyms. The primary sources of material for this checklist are the following collections:

KUMN - Museum of Nature, Kharkiv National University, Ukraine;

ZIN - Zoological Institute, Academy of Sciences,

St. Petersburg, Russia;

ZMUN - Zoological Museum, University of Oslo, Norway;

ZSMC - Zoologische Staatssammlung, München, Germany;

DSc - Private collection of S.V. Dedyukhin, Izhevsk, Russia;

FRc - Private collection of R.V. Filimonov, St. Petersburg, Russia;

NVc - Private collection of V.A. Nemkov, Orenburg, Russia;
RAc - Private collection of A.V. Rusakov, Orenburg, Russia.

Abbreviations:

[HN] — homonym name;

[MI] - misidentification;

[MS] - misspelling;

N.R. - Natural Reserve;

N.P. - National Park.

Plotting of territory of Russia and presentation of distributional information. Plotting of European territory was set up by the European Invertebrate Survey (EIS) in "pre-GPS era" to improve the capturing and analysis of primary distribution data for European invertebrates and for alignment of data representation and mapping. The UTM grid and the $50 \times 50 \mathrm{~km}$ square were adopted as most practical [Wells \& Chatfield, 1992]. Plotting of territory of Russia in grid map system, already approached for comparatively small areas, is problematic due to huge territory of Russia. Recent attempt to arrange the coding of Asian part of Russia is confused, when the codes of provinces of European part were unequally coded with three or fore letters [Legalov, 2010]. A new coding system is here 
Table 1. Alphabetic index of the first and second level subdivisions of Russia Таблица 1. Обозначения перовго и второго уровней территориального подразделения России

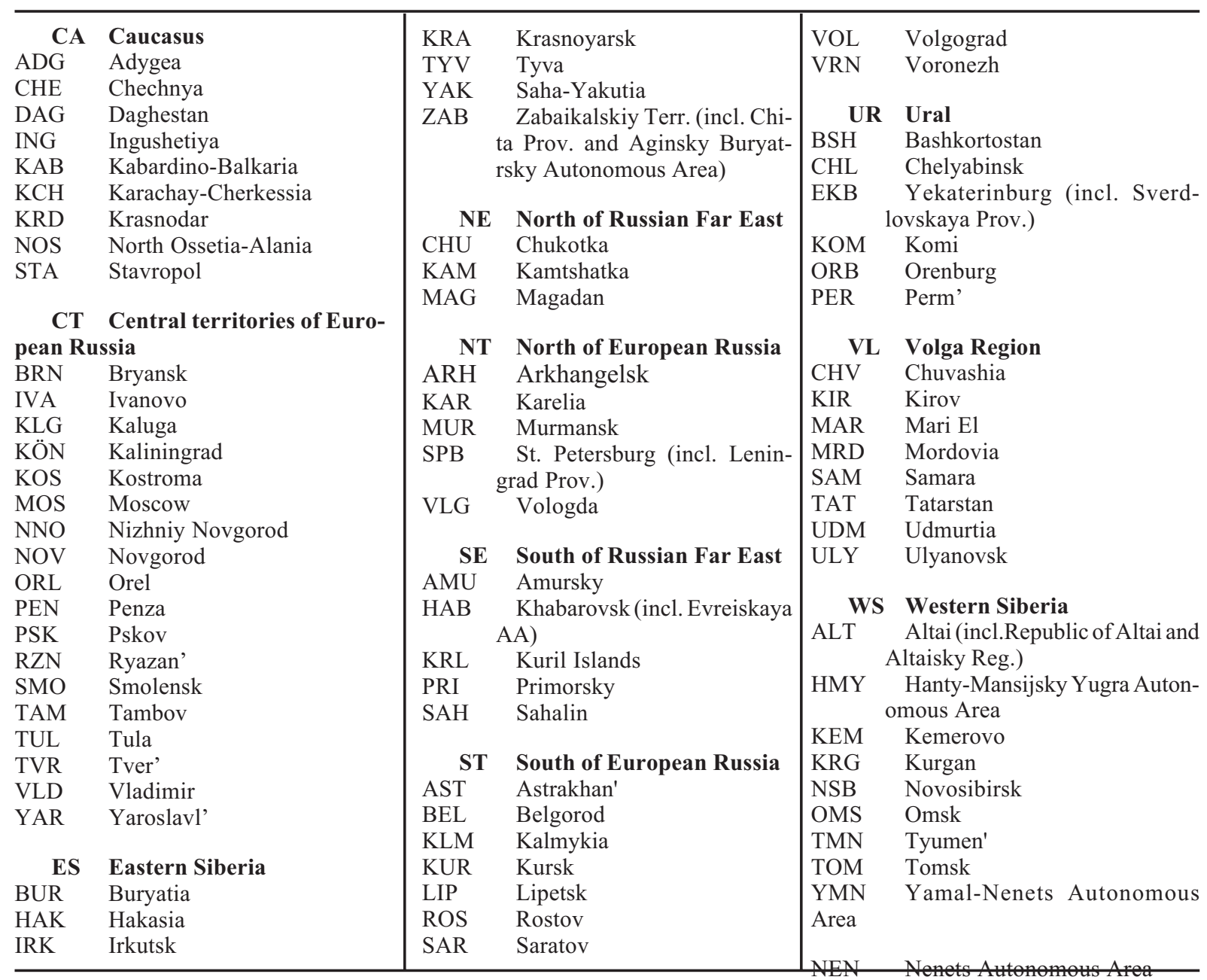

provided, which can be equally applicable for both European and Asian parts of Russia. The first level is the subdivision of Russia (letters in bold) into 10 main parts arranged in geographical order: North of European Russia (NT), Central territories of European Russia (CT), South of European Russia (ST), Caucasus (CA), Volga Region (VL), Ural (UR), Western Siberia (WS), Eastern Siberia (ES), North of Russian Far East (NE), South of Russian Far East (SE). Second level are administrative regions assigned by triple-letter symbols which are arranged in alphabetical order within the first-level subdivision (Table 1).

New records highlighted by asterisk (*). References to records in square brackets [...] are arranged in chronological order. Sources based on original material, dealing with new records and/or providing explicit data on the occurance of species, are only considered. Doubtful records are placed at the end of the checklist. Original material given corresponds mostly to new records and/or additionally for poorly-known species.

\section{Checklist of species occurring in the Volga and Ural Regions}

\section{tribe OTIORHYNCHINI (2 genera, 17 species)}

Otiorhynchus (Altaivagus) unctuosus Germar, 1824

= Otiorhynchus altajensis L. Arnoldi, 1975.

RECORDS.VL: ULY UR: BSH CHL ORB [Isaev, 1990; 1994; Mikhailov, 1999; Dedyukhin, 2011c].

MATERIAL. Orenburg Prov.: 19 (DSc), Donskoye vill., Verblyuzhka Hill, stony steppe, night sweeping, 23.V.2008 (S.V. Dedyukhin); 8ex. (FRc) 14 km NE from Orenburg, Grebeni vill., 13.V.2007 (R.V. Filimonov); 19 (FRc) Sakmara distr., Mayorskoje vill., 24.V.2007 (R.V. Filimonov); 2 + (FRc) Sol'-Iletsk distr., Ishkargan riv., 12.VI.1999 (A.V. Rusakov); Chelyabinsk Prov.: 2 ㅇ (FRc) Kartaly distr., Varshavka vill., on Chartolepis intermedia, 13.VII.1998 (R.V. Filimonov); 5 ㅇ (FRc) Chesma distr., Chernoborsky vill., 7-20.V.2011 (E.A. Chibiliov); Bashkortostan: 5ex. (DSc), Sterlitamak, Shikhan Tratau hill, stony steppe, sweeping at night, 30.V.2010, (S.V. Dedyukhin).

NOTE. O. unctuosus Germar, 1824 was described from "Altai". Its range passes through steppe zone between Volga riv. and Altai. O. minutus and $O$. transparens both were described 
from "Orenburg". According to original descriptions these species have unclear differences from $O$. unctuosus in ratio of size of punctures on pronotal disc and elytral striae; and punctures on elytral intervals. These characters, in $O$. unctuosus, may significantly vary in quite broad limits, and suggest $O$. minutus and $O$. transparens are conspecific with $O$. unctuosus, but types of all these species are to be examined before final conclusion.

\section{Otiorhynchus (Choilisanus) raucus raucus} (Fabricius, 1776)

Curculio raucus Fabricius, 1776.

RECORDS. VL: CHV SAM TAT UDM ULY UR: CHL* ORB* PER [Lindeman, 1871; Lebedev, 1906; Koblova, 1967; Isaev, 1994; 1996; Egorov \& Isaev, 1998; Dedyukhin et al., 2005; Dedyukhin, 2010a; 2011b].

MATERIAL. Chelyabinsk Prov.: 29 (FRc) Ilmen Nature Reserve, 102 forest quarter, pitfall trap, 4-20.VII.2000 (P.V. Rudo-

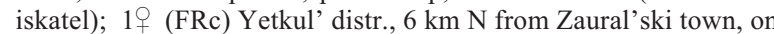
Rumex confertus, 4.VI.1997 (R.V. Filimonov); 1 오 (FRc) Chesma distr., Chernoborskij vill., on Rumex confertus, 29.VI.-2.VII.2011 (R.V. Filimonov); Orenburg Prov.: 1 우 (FRc) Sol'-Iletsk distr., Novoiletsk vill., 8-10.V.2007 (R.V. Filimonov); 1ex. (NVc) Kuvandyk distr., Aituar vill., Orenburgsky N.R.: Aituarskaya Steppe, Shinbutak Ravine, pitfaill traps, 6-11.VII.2001 (V.A. Nemkov).

\section{Otiorhynchus (Choilisanus) velutinus Germar, 1824}

Otiorhynchus humilis Germar, 1824.

RECORDS. VL: CHV TAT MRD SAM UDM ULY UR: CHL EKB PER ORB [Lindeman, 1871 (humilis); Lebedev, 1912; Arnoldi, 1954; Koblova, 1967; Lugovaya, 1970; Isaev, 1994; 1996; Mikhailov, 1999; Database of IEPA, 2009; Dedyukhin, 2006; 2007; 2010a; 2010b; 2011b].

MATERIAL. Orenburg Prov.: 1f (FRc) $14 \mathrm{~km}$ NE from Orenburg, Grebeni vill., 13.V.2007 (R.V. Filimonov); 2f (FRc) Sol'Iletsk distr., Novoiletsk vill., 25-27.V.2008 (R.V. Filimonov).

\section{Otiorhynchus (Choilisanus) pilosus Gyllenhal, 1834 \\ = Otiorhynchus caucasicus Stierlin, 1872.}

RECORDS. VL: SAM ULY [Isaev, 1994;1996; 2007].

\section{Otiorhynchus (Cryphiphorus) ligustici (Linnaeus,} 1758) (Curculio)

Curculio ligustici Linnaeus, 1758.

RECORDS. VL: CHV KIR MRD SAM TAT UDM ULY UR BSH CHL EKB ORB PER* [Lindeman, 1871; Csiki, 1901; Lebedev, 1906; Jakovlev, 1910; Koblova, 1967; Lugovaya, 1970; Shernin, 1974; Arnoldi et al., 1974; Esyunin \& Kozminykh, 1992; Isaev, 1994; 1996; Lagunov \& Novozhenov, 1996; Dedyukhin et al., 2005; Tshitshikov \& Legalov, 2008; Database of IEPA, 2009; Dedyukhin, 2010d]

MATERIAL. Perm' Reg.: 1ex. (DSc) Kungur, Spasskaya Mt., stony steppe, 16.VII.2009 (S.V. Dedyukhin); 1 ex. (DSc), Dobryansky distr., Lunezhki vill., on Cirsium setosum, 2.VI.2010(S.V. Dedyukhin); Orenburg Prov.: $1+(\mathrm{NVc})$ Belyaevka distr., Orenburgsky N.R.: Burtinskaya Steppe, pitfall trap, 5.VII.2006 (V.A. Nemkov).

\section{Otiorhynchus (Microphalantus ) arcticus (Fabricius,} 1780)

Curculio arcticus Fabricius, 1780.

RECORDS. UR: EKB KOM* [Arnoldi et al. 1965].

MATERIAL. Komi: 1ex. (ZIN) "middle course of Petshera", 1906 (P. Ivanov).

NOTE. Record from KIR by Jakovlev [1910] and Shernin [1974] was based on mislabeled specimens.

Otiorhynchus (Nihus) scaber (Linnaeus, 1758)

Curculio scaber Linnaeus, 1758.

RECORDS. VL: MAR* MRD TAT [Aleynikova, 1964].

MATERIAL. Mari El: 39 (ZIN) Gornomariysky distr., $95 \mathrm{~km} \mathrm{~W}$ of Yoshkar-Ola, near Karas'yar Lake, N56 35' 36' 'E46 21' 18', 26.VII.2006 (N.N. Yunakov).

\section{Otiorhynchus (Otiolehus) tristis (Scopoli, 1763)}

Curculio tristis Scopoli, 1763.

RECORDS. VL: CHV KIR MRD SAM TAT UDM ULY UR: BSH* CHL KOM ORB* PER* [Lebedev, 1906; Jakovlev, 1910; Lugovaya, 1970; Shernin, 1974; Roschinenko, 1972; Isaev, 1994; 1996; Lagunov \& Novozhenov, 1996; Dedyukhin et al., 2005; Dedyukhin, 2010d; Dedyukhin \& Tselischeva, 2011].

MATERIAL. Perm' Reg.: 1 ex. (DSc), Kungur distr., Kungur, Spasskaya Mt., on Inula salicina, 16.VII.2009 (S.V. Dedyukhin); 1 ex. (DSc), Gorbunyata vill., limestone-gypseous slope, 14.VII.2009 (S.V. Dedyukhin); 1ex. (DSc), Nytvensky distr., Sosnova vill., on Cirsium setosum, 29.VI.2010 (S.V. Dedyukhin); Bashkortostan: 5ex. (DSc), Sterlitamak, Shikhan Tratau, steppe, sweeping at night, 30.V.2010 (S.V. Dedyukhin) ); Orenburg Prov.: 2 ㅇ (FRc) Sakmara distr., Mayorskoje vill., 24.V.2007 (R.V. Filimonov); 19 (FRc) idem, 1.VI.2008 (R.V. Filimonov).

Otiorhynchus (Otiorhynchus) concinnus Gyllenhal, 1834

= Otiorhynchus steppensis Faust, 1888 .

RECORDS. VL: SAM* TAT UR: BSH* CHL* ORB [Faust, 1888; Lebedev, 1906 (borealis)].

MATERIAL. Samara Prov.: 19 (ZIN) "Samara"; Orenburg Prov.: $20^{7} 29$ (FRc) $14 \mathrm{~km}$ NE from Orenburg, Grebeni vill., 24.V.2006 (R.V. Filimonov); 1 \% (FRc) idem, 27.V.2007 (R.V. Filimonov); $1 \sigma^{7} 3$ (FRc), Belyaevka distr., Donskoye vill., Verblyuzhka Hill, 28-29.V.2006, (R.V. Filimonov); $10^{\text {T }}$ (FRc) Kuvandyk distr., Maloye Churaevo vill., Shaitantau ridge, 26-28.V.2010, (A.M. Shapovalov); 6 + (FRc) idem, 23-28.V.2011 (R.V. Filimonov); Chelyabinsk Prov.: $1 \sigma^{\prime}$ (ZIN) "Oberlauf des Ural, Kisilskaja" [no date] (E. v. Bodemeyer); 1 + (FRc) Chesma distr., Chernoborskij vill., 7-20.V.2011 (E.A. Chibiliov); $10^{7} 3$ (FRc), idem, 2225.VI.2011 (E.A. Chibiliov \& R.V. Filimonov); Bashkortostan: 1 + (ZIN) Kumertau distr., Yermolaevo vill., 6.VI.1969 (Gilarov); 5ex. (DSc), Sterlitamak, Shikhan Tratau, steppe, sweeping at night, 30.V.2010 (S.V. Dedyukhin)

Otiorhynchus (Pendragon) ovatus (Linnaeus, 1758)

Curculio ovatus Linnaeus, 1758.

RECORDS. VL: CHV KIR MAR* MRD SAM TAT UDM ULY UR: BSH CHL EKB KOM ORB* PER [Lindeman, 1871; Csiki, 1901; Lebedev, 1906; Jakovlev, 1910; Chetyrkina, 1926; Lugovaya, 1970; Roschinenko, 1981; Isaev, 1994; 1996; Lagunov \& Novozhenov, 1996; Ermakov, 2003; Dedyukhin et al., 2005; Tshitshikov \& Legalov, 2008; Database of IEPA, 2009; Dedyukhin \& Tselischeva, 2011].

MATERIAL. Mari El: 10 (ZIN) Gornomariisky distr., $90 \mathrm{~km}$ WSW of Yoshkar-Ola, $16 \mathrm{~km}$ NE of Yurkino vill., nr Karasjar Lake, 102 m, N56³6' 42.8' ' E46²3' 37.5' 28.VII.2005 (N.N. Yunakov); $2+$ (ZIN) Medvedevo distr., Bolshaya Kokshaga N.R., nr Shaptunga vill., $150 \mathrm{~m}, \mathrm{~N} 56^{\circ} 38^{\prime} 31.92^{\prime \prime}$ E47 $13^{\prime}$ 45.41' meadow, sweeping, 29.VII.2006 (N.N. Yunakov); Orenburg Prov.: 1 우 (FRc) Sakmara distr., Mayorskoje vill., 24.V.2007 (R.V. Filimonov); 5 (FRc) Sol'-Iletsk distr., Novoiletsk vill., 8-10.V.2007 (R.V. Filimonov).

\section{Otiorhynchus (Phalantorrhynchus) politus Gyllenhal 1834}

RECORDS. VL: SAM UDM UR: BSH CHL EKB KOM* [Isaev, 1996; Ermakov, 2003; Dedyukhin et al., 2005; Database of IEPA, 2009; Dedyukhin, 2011c].

MATERIAL. Bashkortostan: 1ex. (ZIN) Birsk, 1918 (Kossakovsky); 4ex. (ZIN) Irgizla, 12-28.VI.1899 (Jacobson \& Schmidt); $2 \sigma^{7} 1$ 우 (FRc) Mt. Iremel, 1000-1300 m, 1-4.VI.2000 (R.V. Filimonov); Komi: 150ex. (ZIN) Ust-Tsilma vill., 8.V.1906 (A.V. Zhuravsky); 12ex. (ZIN) Adzva vill., 21.VII.1909 (A.V. Zhuravsky).

Otiorhynchus (Podoropelmus) fullo (Schrank, 1781)

Curculio fullo (Schrank, 1781).

= Curculio zebra (Fabricius, 1792).

RECORDS. VL: CHV MRD SAM TAT ULY UR: ORB [Lindeman, 1871 (zebra); Koblova, 1967; Lugovaya, 1970; Egorov \& Isaev, 1998; Arnoldi et al., 1974; Isaev, 1994; 1996]. 
MATERIAL. Orenburg Prov.: 1ex. (NVc) Belyaevka distr., Orenburgsky N.R.: Burtinskaya Steppe, Karmen Mt., pitfall traps, 17-21.VIII.2000 (V.A. Nemkov); 1 ex. (NVc) Kuvandyk distr. Aituar vill., Orenburgsky N.R.: Aituarskaya Steppe, Shinbutak Ravine, pitfaill traps, 6-11.VII.2001 (V.A. Nemkov); 1ex. (NVc) idem, 5-10.VII.2002 (V.A. Nemkov).

Otiorhynchus (Podoropelmus) scopularis Hochhuth, 1847

RECORDS. VL: SAM TAT UDM ULY UR: ORB [Lebedev, 1906; Dmitriev, 1935; Isaev, 1994; 1996; Dedyukhin et al., 2005; Dedyukhin, 2003; 2006; 2009; 2010b; 2011b]/

MATERIAL. Udmurtia: 1ex. (DSc) Zav'yalovsky distr., Doksha vill., Kama riv. bank, under Ulmus bark, 24.IV.2001 (S.V. Dedyukhin); 2ex. (DSc) Votkinskiy distr., Sidorovy Gory vill., Kama riv, bank, on microsporangium of Pinus sylvestris., 31.V.1996 (S.V. Dedyukhin), 1ex. (DSc), idem, 22.IX.2007, under stone (S.V. Dedyukhin); 1ex. (DSc) Sarapulsky distr., Netshkino vill., Kama riv. bank, oak forest, under bark, 25.IX.2008 (S.V. Dedyukhin); 1ex. (DSc) Karakulinsky distr., Ust'-Be'sk vill., Kama riv. bank, under stone, 10.V.2007 (S.V. Dedyukhin), 1ex. (DSc) idem, Bolshaya Yemasha riv. meadow near nemoral forest, 17.VI.2003 (S.V. Dedyukhin); Tatarstan: 5ex. (DSc), Agryz distr., Krasny Bor town, Kama riv. bank, pine forest, on Cotoneaster melanocarpus, 31.V.2009 (S.V. Dedyukhin); Orenburg Prov.: 2 (ZIN) Tashla distr., Miroshkino vill., 31.V.1950 (Martynova); 1ex. (NVc) Novosergievka distr., Staraya Belogorka vill., 14 20.VI.2008 (V.A. Nemkov).

Otiorhynchus (Postaremus) nodosus (Müller, 1764)

Curculio nodosus Müller, 1764.

= Otiorhynchus dubius Ström, 1765.

= Otiorhynchus maurus Schrank 1781

RECORDS. VL: KIR TAT UDM UR: BSH CHL EKB KOM PER [Lebedev, 1906; Jakovlev, 1910; Shernin, 1974; Sedykh, 1974; Shernin, 1974; Ermakov, 2003; Database of IEPA, 2009; Dedyukhin, 2011c]

MATERIAL. Bashkortostan: $40^{T} 3+$ (FRc) Mt. Iremel, 10001300 m, on Aconogonon alpinum, 1-4.VI.2000 (R.V. Filimonov); idem, 8.VII.1999 (E.V. Guskova).

Otiorhynchus (Proremus) smreczynskii Cmoluch, 1961

RECORDS. VL: CHV ULY UR: CHL* ORB* [Isaev, 1990; 1994; Egorov \& Isaev, 1998; Isaev et al., 2004].

MATERIAL. Chelyabinsk Prov.: 5 ㅇ (FRc) Chelyabinsk, Fedorovka suburb, 31.V.2009 (R.V. Filimonov); 70 ㅇ (FRc) idem, in lea litter under Syringa vulgaris, 1.VI.2009 (R.V. Filimonov); 3ㅇ (FRc) idem, in leaf litter under Lonicera tatarica, 3.VI.2009 (R.V. Filimonov); Orenburg Prov.: 1ex. (NVc) Orenburg, Rostoshi suburb, 30.V.2007 (V.A. Nemkov).

Otiorhynchus (Pseudocryphiphorus) conspersus (Herbst, 1795)

Curculio conspersus Herbst, 1795.

RECORDS. VL: CHV MRD SAM TAT* ULY UR: BSH* CHL ORB [Lindeman, 1871; Dmitriev, 1935; Koblova, 1967; Lugovaya 1970; Esyunin \& Kozminykh, 1992; Isaev, 1994; 1996; Mikhailov, 1999; Egorov, Nikolaeva, 2005]

MATERIAL. Tatarstan: 1ex. (DSc) Leninogorsky distr., Staraya Pismyanka vill., stony steppe, 28.IV.2009 (S.V. Dedyukhin); Baschkortostan: 1ex. (DSc) Sterlitamak, Shikhan Tratau hill, stony steppe, sweeping at night, 30.V.2010 (S.V. Dedyukhin); Orenburg Prov.: 3ex. (NVc) Belyaevka distr., Orenburgsky N.R.: Burtinskaya Steppe, 17-20.VII.1991 (V.A. Nemkov); 1ex. (NVc) idem, 6.V.1993 (V.A. Nemkov); 1ex. (NVc) Kuvandyk distr., Aituar vill., Orenburgsky N.R.: Aituarskaya Steppe, Shinbutak Ravine, 25-30.VI.1991 (V.A. Nemkov); 1ex. (NVc) idem, 25-31.V.2007 (V.A. Nemkov); 1 우 (FRc) Sol'-Iletsk distr., Novoiletsk vill., 25-27.V.2008 (A.M. Shapovalov)

Parameira (Parameira) gebleri Faust, 1893

= Parameira volgense Korotyaev, 1992.

RECORDS. VL: ULY UR: CHL [Korotyaev, 1992; Isaev, 1994; Mikhailov, 1999]. tribe PERITELINI (2 genera, 2 species)

Centricnemus leucogrammus (Germar, 1824)

Peritelus leucogrammus Germar, 1824.

RECORDS. VL: CHV SAM ULY UR: BSH CHL [Isaev, 1994; 1996; Egorov \& Isaev, 1998; Mikhailov, 1999; Tshaschina, 2002; Dedyukhin, 2010c]

Peritelus familiaris Boheman, 1834

RECORDS. VL: TAT [Csiki, 1901].

tribe TRACHYPHLOEINI (4 genera, 6 species)

Trachyphloeus (Trachyphloeus) parallelus Seidlitz, 1868 RECORDS. VL: TAT* UR: ORB*.

TYPE MATERIAL. Tatarstan: 1 ( (ZSMC) "Kasan"; 1 iq syntype (ZSMC) "Ullr." "341", Type von / Trachyphloeus parallelus Seidl.

MATERIAL. Tatarstan: 1ㅇ (ZSMC) "Kasan"; 1우 (ZSMC) "Ullr.", "341", Type von / Trachyphloeus parallelus Seidl.; 2 \% (KUMN) "Kazan, Lebedev"; Orenburg Prov.: 3ex. (NVc) Tashla distr., Trudovoye vill.(V.A. Nemkov); 3ex. (NVc) Belyaevka distr., Orenburgsky N.R.: Burtinskaya Steppe (V.A. Nemkov); 1 ex. (NVc) Kuvandyk distr., Aituar vill., Orenburgsky N.R.: Aituarskaya Steppe, Shinbutak Ravine (V.A. Nemkov).

\section{Trachyphloeus (Trachyphloeus) scabriculus} (Linnaeus, 1771)

Curculio scabriculus Linnaeus, 1771.

RECORDS. VL: TAT ULY [Lebedev, 1912; Isaev, 2000].

\section{Trachyphloeus (Trachyphloeus) spinimanus Germar, 1824}

RECORDS. VL: CHV TAT SAM ULY UR: BSH* CHL ORB PER [Csiki, 1901; Lebedev, 1906; Esyunin \& Kozminykh, 1992; Isaev, 1994; 1996; Egorov \& Isaev, 1998; Dedyukhin, 2010a; 2011b].

MATERIAL. Bashkortostan: 3ex. (DSc) Sterlitamak, Shikhan Yuraktau hill, stony steppe, 29.V.2010 (S.V. Dedyukhin).

Romualdius bifoveolatus (Beck, 1817)

Curculio bifoveolatus Beck, 1817.

RECORDS. VL: CHV KIR MRD UDM ULY UR: ORB* [Isaev, 1994 (Trachyphloeus); Egorov \& Isaev, 1998 (Trachyphloeus); Yuferev, 2001a (Trachyphloeus); Dedyukhin et al., 2005 (Trachyphloeus)].

MATERIAL. Orenburg Prov.: 1ex. (NVc) Pervomaysky distr., Orenburgsky N.R.: Talovskaya Steppe (V.A. Nemkov); 2ex. (NVc) Belyaevka distr., Orenburgsky N.R.: Burtinskaya Steppe (V.A. Nemkov).

Stuebenius amplithorax (Formánek, 1907)

Trachyphloeus amplithorax Formánek, 1907.

RECORDS. UR: ORB*.

MATERIAL. Orenburg Prov.: 6ex. (FRc) $20 \mathrm{~km} \mathrm{SSW} \mathrm{from}$ Orenburg, Donguz river valley, under leaves of Verbascum phoeniceum L., 17.V.2007 (R.V. Filimonov); 2ex. (FRc) idem, pitfall trap, 11-20.V.2007 (V.O. Kozminykh).

Cathormiocerus aristatus (Gyllenhal, 1827)

Trachyphloeus aristatus Gyllenhal, 1827.

RECORDS. VL: CHV TAT MRD SAM UDM ULY UR: CHL KOM ORB* PER [Lebedev, 1906; Baskina, Friedman 1928; BeiBienko, 1946; Lugovaya, 1970; Isaev, 1994; 1996; Egorov \& Isaev, 1998; Dedyukhin et al., 2005].

MATERIAL. Chelyabinsk Prov.: 19 (FRc) Ilmen Nature Reserve, 73 forest quarter, pitfall trap, 15-14.VII.2000 (P.V. Rudoiskatel); 1 ( (FRc) Ilmen Nature Reserve, 102 forest quarter, pitfall trap, 20.VII.-6.VIII.2000 (P.V. Rudoiskatel); 19 (FRc) $22 \mathrm{~km} \mathrm{E}$ from Chelyabinsk, Sykandyk lake, 6.V.2000 (R.V. Filimonov); Orenburg Prov.: 1 (FRc) 8 ( $\mathrm{NVc}$ ) Belyaevka distr., Orenburgsky N.R.: Burtinskaya Steppe, 18-24.VIII.2009 (V.A. Nemkov); 1 웅 $(\mathrm{NVc})$ Kuvandyk distr., Aituar vill., Orenburgsky N.R.: Aituarskaya Steppe (V.A. Nemkov). 
tribe CYPHICERINI (3 genera, 3 species)

Ptochus (Ptochus) porcellus Boheman, 1834

RECORDS. UR: ORB [Arnoldi, 1954].

MATERIAL. Orenburg Prov.: 1ex. (FRc) Akbulak distr., Akoba vill., 11.VI.1990 (A.V. Rusakov); 1ex. (FRc) Sakmara distr. Mayorskoje vill., 1.VI.2008 (R.V. Filimonov); 5ex. (FRc) Kuvandyk distr., Aituar vill., Orenburgsky N.R.: Aituarskaya Steppe, 18.VIII.2006 (K.A. Khristina); 1ex. (FRc) Sol-Iletsk distr., Troitsk vill., steppe on chalky hills, 8-22.VI.2008 (V.A. Nemkov); 1ex. (DSc) Donskoye vill., Verblyuzhka Hill, stony steppe, sweeping at night, 23.V.2008 (S.V. Dedyukhin); 1ex. (DSc) idem, 29.VI.2008 (S.V. Dedyukhin).

Sphaeroptochus deportatus (Boheman, 1834)

Ptochus eportatus Boheman, 1834.

RECORDS. UR: ORB*.

MATERIAL. Orenburg Prov.: 3ex. (FRc) Gai distr., Kazachya Guberla vill., on Caragana frutex (L.) C. Koch, 18.V.2006 (R.V. Filimonov); 3ex. (FRc) Gai distr., Guberla vill., steppe, 20.VII.2006 (V.A. Nemkov); 1 ex. (FRc) Kuvandyk distr., Aituar vill., Orenburgsky N.R.: Aituarskaya Steppe, 29.V.2009 (V.A. Nemkov); 2ex (DSc) Donskoye vill., Verblyuzhka Hill, stony steppe, sweeping at night, 23.V.2008 (S.V. Dedyukhin).

\section{Chloebius immeritus Boheman, 1826}

RECORDS. UR: ORB*

MATERIAL. Orenburg Prov.: 30ex. (FRc) Sol'-Iletsk distr., Novoiletsk vill., 25-27.V.2008 (R.V. Filimonov); $2 O^{7}$ (FRc) Akbulak distr., Akoba vill., 21-23.VI.2007 (A.M. Shapovalov).

tribe PHYLLOBIINI (1 genus, 16 species)

Phyllobius (Alsus) brevis Gyllenhal, 1834

RECORDS. VL: CHV KIR MRD SAM TAT UDM ULY UR: BSH* CHL EKB ORB* PER [Csiki,1901; Lebedev, 1906 (sulcirostris); Jakovlev, 1910 (gyrates); Koblova, 1967; Lugovaya, 1970 Roschinenko, 1981; Isaev, 1994; 1996; Mikhailov, 1999; Dedyukhin et al., 2005; Tshitshikov \& Legalov, 2008; Dedyukhin \& Tselischeva, 2011; Dedyukhin, 2011b]

MATERIAL. Bashkortostan: 5ex. (DSc) Sterlitamak, Shikhan Tratau hill, 31.V.2010 (S.V. Dedyukhin); Orenburg Prov.: 10, 1우 (FRc) Belyaevka distr., Orenburgsky N.R.: Burtinskaya Steppe, 26.V.2007 (V.A. Nemkov); 2ex. (RAc) Buzuluk distr., Buzulukskij Bor N.P., 6.VI.2007 (K.A. Khristina)

\section{Phyllobius (Dieletus) argentatus (Linnaeus, 1758)}

Curculio argentatus Linnaeus, 1758

RECORDS. VL: CHV KIR MRD SAM TAT UDM ULY UR: BSH CHL EKB KOM ORB [Lebedev, 1906; Polozhentsev, 1947; Lugovaya, 1970; Shernin, 1974; Isaev, 1994; 1996; Tshaschina, 2002; Ermakov, 2003; Dedyukhin et al., 2005; Database of IEPA, 2009; Dedyukhin \& Tselischeva, 2011].

MATERIAL. Orenburg Prov.: 1ex. (NVc) 1 (오요 (FRc) Novosergievka distr., Staraya Belogorka vill., 14.VI.2008 (V.A. Nemkov); $20^{7} 3$ (FRc) $8 \mathrm{~km}$ E from Mednogorsk, Blyava vill., 12.VI.2009, (R.V. Filimonov).

\section{Phyllobius (Metaphyllobius) dahli Korotyaev, 1984}

RECORDS. VL: CHV KIR UDM ULY UR: ORB [Korotyaev, 1984; Yunakov \& Korotyaev, 2007; Dedyukhin, 2010b; 2011a; Dedyukhin \& Tselischeva, 2011].

MATERIAL. Udmurtia: 2ex. (DSc) Kambarsky distr., Kambarka town, on Artemisia abrotanum and Tanacetum vulgare, sweeping, 19.VII.2003; 1ex. (DSc) idem, on Ptarmica salicifolia, sweeping, 2.VII.2010 (S.V. Dedyukhin); Kirov Prov.: 1ex. (DSc) Sovetsky distr., Tyaptichi vill., Nemda riv. bank, 1.VI.2010 (L.G. Tselischeva); $10^{\top}$ (DSc) Kotel'nitsh distr., Nurgush N.R., Salix shrubs near Vyatka riv., pitfall traps, 10-21.VI.2008 (L.G. Tselischeva); $1 \mathrm{O}^{7}$ (DSc) Kotel'nitsh Vyatka riv. bank, on Ptarmica salicifolia, 12.VII.2010 (S.V. Dedyukhin); 1ex. (DSc) Lebyazhsky distr., Priverkh vill., Vyatka riv. bank, on Artemisia abrotanum, sweeping, 26.VI.2010 (S.V Dedyukhin); $20^{7} 1$ 우 (DSc) Kirov, Vyatka riv. flood land, on Salix acutifolia, 16.VI.2011 (S.V. Dedyukhin); Orenburg Prov.: 1 1 (FRc) 8 km E from Mednogorsk, Blyava vill., 12.VI.2009 (R.V. Filimonov); $20^{7} 5$ ㅇ (FRc) Sol'-Iletsk distr., Novoiletsk vill., Ilek riv., 25-27.V.2008 (R.V. Filimonov \& A.M. Shapovalov).

Phyllobius (Metaphyllobius) glaucus (Scopoli, 1763)

Curculio glaucus Scopoli, 1763.

= Curculio calcaratus (Fabricius, 1792).

= Phyllobius maculatus (Tournier, 1877) [syn. after: Wanat, 2005]

RECORDS. VL: CHV KIR MRD TAT UDM UR: BSH* CHL ORB* PER [Lindeman, 1871 (calcaratus); Csiki, 1901; Lebedev, 1906; Jakovlev, 1910; Lugovaya, 1970; Shernin, 1974; Esyunin \& Kozminykh, 1992 (calcaratus); Lagunov \& Novozhenov, 1996 (calcaratus); Dedyukhin et al., 2005; Yunakov, Korotyaev 2007; Dedyukhin, 2010b; Dedyukhin \& Tselischeva, 2011].

MATERIAL. Bashkortostan: $10^{7} 1$ 우 (FRc) Burzyansky distr., Shulgan-Tash N.R., 6.VI.2000 (P.V. Rudoiskatel); Orenburg Prov.: $10^{7}$ (FRc) Tashla distr., Trudovoye vill., 12.VI.2008 (V.A. Nemkov).

\section{Phyllobius (Metaphyllobius) jacobsoni Smirnov, 1915 stat. res.}

RECORDS. VL: CHV KIR MRD UDM ULY UR: KOM [Lebedev, 1906; Jakovlev, 1910; Roschinenko, 1972; 1981; Shernin, 1974; Isaev, 1990; 1994; 1996; Dedyukhin, 2003; Dedyukhin et al., 2005; Dmitrieva, 2005 (maculatus); Yunakov \& Korotyaev, 2007 (maculatus)].

NOTE. This species was mistakenly synonymized with Ph. maculatus (Tournier, 1877) ( $=$ Ph. glaucus Scopoli, 1763). For diagnosis see [Yunakov \& Korotyaev, 2007].

\section{Phyllobius (Metaphyllobius) pomaceus pomaceus} Gyllenhal, 1834

$=$ Curculio urticae (De Geer, 1775) $[\mathrm{HN}]$ nec Scopoli, 1763.

$=$ Curculio alneti (Fabricius, 1792) [HN] nec Schrank, 1781.

RECORDS. VL: CHV KIR MAR MRD SAM TAT UDM ULY UR: BSH CHL EKB KOM ORB PER [Lindeman, 1871 (alneti); Csiki, 1901 (urticae); Lebedev, 1906; Jakovlev, 1910; Lugovaya, 1970 (urticae); Shernin, 1974; Roschinenko, 1972; Isaev, 1994; 1996; Lagunov \& Novozhenov, 1996 (urticae); Dedyukhin et al., 2005; Yunakov \& Korotyaev, 2007; Tshitshikov \& Legalov, 2008; Database of IEPA, 2009; Dedyukhin, 2010d; Dedyukhin \& Tselischeva, 2011].

MATERIAL. Orenburg Prov.: $2 \sigma^{\top} 4+$ (FRc) Tyulgan distr., Tashla vill., 29-31.V.2006 (V.E. Grigorjev); 19 (FRc) Gai distr., Kazachya Guberla vill., 18.V.2006 (R.V. Filimonov).

Phyllobius (Nanoschetus) cylindricollis Gyllenhal, 1834

RECORDS. VL: CHV UR: CHL ORB* [Esyunin \& Kozminykh, 1992; Egorov, 2004].

MATERIAL. Orenburg Prov.: 3ex. (NVc) Tashla distr., Trudovoye vill. (V.A. Nemkov); $60^{7} 3$ ? (FRc) Svetly distr., Bolshoy Obalykol' Lake, 5.VI.2006 (R.V. Filimonov); $80^{7} 3$ (FRc) Svetly distr., Karakol' Lake, on Poaceae g.sp., 7.VI.2006 (R.V. Filimonov).

Phyllobius (Nemoicus) oblongus (Linnaeus, 1758)

Curculio oblongus Linnaeus, 1758

RECORDS. VL: CHV KIR MRD SAM TAT UDM ULY UR: BSH CHL EKB ORB [Lebedev,1906; Jakovlev, 1910; Lugovaya, 1970; Shernin, 1974; Isaev, 1994; 1996; Dedyukhin et al., 2005; Dedyukhin, 2010d; Dedyukhin \& Tselischeva, 2011].

MATERIAL. Bashkortostan: $2+$ (FRc) Burzyansky distr., Akbulatovo-Mulatovo vill., 5-6.VI.2000 (P.V. Rudoiskatel); Chelyabinsk Prov.: 1 우 (FRc) Chebarkul distr., Kisegach railway station, 21.VI.2000 (E.V. Guskova); 1 ( (FRc) Chelyabinsk, Fedorovka suburb, on Malus domestica, 15.VI.2008 (R.V. Filimonov).

Phyllobius (Parnemoicus) viridicollis (Fabricius, 1792)

Curculio viridicollis Fabricius, 1792.

RECORDS. VL: CHV TAT UDM ULY UR: BSH CHL EKB ORB [Lindeman, 1871; Csiki,1901; Lebedev, 1906; 1912; Isaev, 
1994; Lagunov \& Novozhenov, 1996; Egorov \& Isaev, 1998; Dedyukhin et al., 2005; Dedyukhin, 2003; 2009; Database of IEPA, 2009].

MATERIAL. Bashkortostan: 39 (FRc) Mt. Iremel, 8.VII.1999 (E.V. Guskova); 1 으 (FRc) Burzyansky distr., Shulgan-Tash Na-

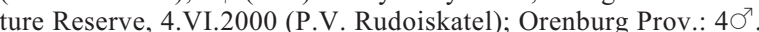
1 우 (FRc) Kuvandyk distr., Maloye Churaevo vill., Shaitantau ridge, 10.VI.2009 (R.V. Filimonov).

Phyllobius (Phyllobius) arborator (Herbst, 1797)

Curculio arborator Herbst, 1797.

= Phyllobius psittacinus Germar, 1824 .

RECORDS. VL: CHV KIR MRD TAT UDM UR: CHL EKB [Lindeman, 1871 (psittacinus); Lebedev, 1906 (psittacinus); Jakovlev, 1910 (psittacinus); Shernin, 1974 (psittacinus); Esyunin \& Kozminykh, 1992; Lagunov \& Novozhenov, 1996; Egorov \& Isaev, 1998; Dedyukhin et al., 2005; Database of IEPA, 2009].

\section{Phyllobius (Phyllobius) pyri (Linnaeus, 1758)}

Curculio pyri Linnaeus, 1758.

= Curculio vespertinus (Fabricius, 1792).

RECORDS. VL: CHV KIR MRD SAM TAT UDM ULY UR: BSH* CHL EKB KOM ORB PER [Lindeman, 1871; Lebedev, 1906; Jakovlev, 1910; Bei-Bienko, 1946; Koblova, 1967; Lugovaya, 1970; Roschinenko, 1972; Shernin, 1974; Esyunin \& Kozminykh, 1992; Isaev, 1994; 1996; Lagunov \& Novozhenov, 1996; Mikhailov, 1999; Yuferev, 2001 (vespertinus); Ermakov, 2003; Tshitshikov \& Legalov, 2008; Database of IEPA, 2009; Dedyukhin et al., 2005; Dedyukhin, 2010d; Dedyukhin \& Tselischeva, 2011; Dedyukhin et al., 2005].

MATERIAL. Bashkortostan: 5ex. (FRc) Burzyansky distr., Shulgan-Tash Nature Reserve, 3.VI.2000 (P.V. Rudoiskatel); Orenburg Prov.: 1ex. (RAc) Buzuluk distr., Buzulukskij Bor N.P., 12.V.2007 (K.A. Khristina); 1ex. (NVc) Tashla distr., Trudovoye vill., 1013.V.2007(V.A. Nemkov); 1ex. (NVc)idem, 12.VI.2008(V.A. Nemkov)

Phyllobius (Pterygorrhynchus) contemptus Steven, 1829

RECORDS. VL: CHV MRD SAM TAT* UDM ULY UR: BSH* CHL ORB* PER [Lugovaya, 1970; Isaev, 1994; 1996; Egorov \& Isaev, 1998; Dedyukhin et al., 2005; Dedyukhin, 2003; 2011b; Tshitshikov \& Legalov, 2008].

MATERIAL. Tatarstan: 6ex. (Dsc) Agryz distr., Sukman vill., dry meadow, 30.VI.2001 (S.V. Dedyukhin); Bashkortostan: 1ex. (DSc) Sterlitamak, Shikhan Tratau hill, 31.V.2010 (S.V. Dedyukhin).

\section{Phyllobius (Pterygorrhynchus) crassipes Motschulsky, 1860}

RECORDS. VL: CHV? UDM SAM UR: ORB [Faust, 1881; Lona 1938; Roschinenko, 1972; Egorov \& Isaev, 2005].

MATERIAL. Orenburg Prov.: $10^{7}$ (FRc) Sakmara distr., Donskoje vill., 27.V.2006 (V.E. Grigorjev); 40', 7 ㅇ (FRc) Sol'-Iletsk distr., Novoiletsk vill., Ilek riv., 25-27.V.2008 (R.V. Filimonov); 1 ex. (DSc) Donskoye vill., Verblyuzhka Hill, Ural riv. flood-land, 23.V.2008 (S.V. Dedyukhin).

\section{Phyllobius (Pterygorrhynchus) maculicornis Germar, 1824}

RECORDS. VL: CHV KIR MRD SAM TAT UDM ULY UR: BSH* CHL ORB* PER* [Lindeman, 1871; Lebedev, 1906; Jakovlev, 1910; Lugovaya, 1970; Roschinenko, 1972; Shernin, 1974; Isaev, 1994; 1996; Mikhailov, 1999; Dedyukhin et al., 2005; Dedyukhin \& Tselischeva, 2011].

MATERIAL. Bashkortostan: 5ex. (DSc) Sterlitamak, Shikhan Tratau hill, steppe, on Polygonum alpinum, 30.V.2010 (S.V. Dedyukhin); Orenburg Prov.: 1ex. (RAc) Tyulgan distr., Tashla vill., 30.V.2006 (A.V. Rusakov); $20^{7}, 2$ (FRc) idem, 29-31.V.2006 (V.E. Grigorjev); Perm' Reg.: 3ex. (DSc) Solikamsk distr., Tyulkino vill., Kama riv. bank, on Salix viminalis, 21.VI.2011 (S.V. Dedyukhin).

\section{Phyllobius (Subphyllobius) thalassinus Gyllenhal, 1834}

$=$ Phyllobius scutellaris Redtenbacher, 1849 .

= Phyllobius mutabilis Desbrochers, 1873 .
RECORDS. VL: CHV KIR MRD SAM TAT* UDM ULY UR: CHL* KOM ORB* PER* [Csiki,1901 (mutabilis); Shernin, 1974; Isaev, 1994; 1996; Dedyukhin et al., 2005; Dedyukhin, 2009; Dedyukhin \& Tselischeva, 2011].

MATERIAL. Tatarstan: 1ex. (DSc) Mamadysh, Vyatka riv., flood land, 14.VI.2006 (S.V. Dedyukhin); Perm' Reg.: 1ex. (DSc) Tshaikovsky distr. Volkovsky town, Kama riv. flood land, 17.VI.2009 (S.V. Dedyukhin); Chelyabinsk Prov.: 4+ (FRc) Chesma distr., Chernoborskij vill., 22.VI.-2.VII.2011 (R.V. Filimonov); Orenburg Prov.: $1+$ (NVc), 1 우 (FRc) Tashla distr., Trudovoye vill., 12.VI.2008 (V.A. Nemkov).

Phyllobius (Subphyllobius) virideaeris (Laicharting, 1781)

Curculio virideaeris Laicharting, 1781.

= Curculio uniformis (Marsham, 1802).

RECORDS. VL: ULY UR: ORB [Lindeman, 1871 (uniformis); Isaev, 1994; 2000].

MATERIAL. Orenburg Prov.: $2 q$ (FRc), 29 (NVc) Belyaevka distr., Orenburgsky N.R.: Burtinskaya Steppe, 26.V.2007 (V.A. Nemkov \& V.O. Kozminykh) ); 5 ㅇ (FRc) Kuvandyk distr., Aituar vill., Orenburgsky N.R.: Aituarskaya Steppe, 29-31.V.2007 (V.A. Nemkov).

tribe OMIINI (3 genera, 5 species)

Omias murinus (Boheman, 1843)

Mylacus murinus Boheman, 1843.

RECORDS. VL: CHV MRD TAT SAM ULY UR: ORB [Lebedev, 1906; Lugovaya, 1970; Isaev, 1994; 1996].

MATERIAL. Orenburg Prov.: $60^{7} 49$ (FRc) Gai distr., Kazachya Guberla vill., 18.V.2006 (R.V. Filimonov); $80^{7} 6$ (FRc) Sol'Iletsk distr., $5 \mathrm{~km} \mathrm{~S}$ from Pervomaiskoje vill., Shubaragash forest, 18-21.V.2007 (R.V. Filimonov); $60^{7} 7$ ㅇ (FRc) Sol'-Iletsk distr.,

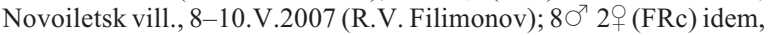
26.V.2008 (R.V. Filimonov).

\section{Omias puberulus Boheman, 1834}

$=$ Curculio rotundatus (Fabricius, 1792).

RECORDS. VL: CHV KIR MRD SAM TAT UDM ULY UR: BSH CHL ORB PER [Lindeman, 1871 (rotundatus); Csiki, 1901; Lebedev, 1906; Jakovlev, 1910; Koblova, 1967 (rotundatus); Lugovaya, 1970 (rotundatus); Esyunin \& Kozminykh, 1992 (rotundatus); Isaev, 1994; 1996; Mikhailov, 1999; Dedyukhin, 2003 (Omiomima rotundata); Dedyukhin et al., 2005; Dedyukhin, 2010a; 2010c; 2011b].

\section{Omias verruca Steven, 1829}

RECORDS. VL: MRD SAM* ULY UR: CHL ORB [Koblova, 1967; Lugovaya, 1970; Isaev, 1994; Mikhailov, 1999].

MATERIAL. Samara Prov.: 1ex. (DSc) Kinelsky distr., Tshubovka vill., stony steppe, 2.V.2010, (S.V. Dedyukhin); Orenburg Prov.: 69 (FRc) Belyaevka distr., Orenburgsky N.R.: Burtinskaya Steppe, 25.V.2007 (V.A. Nemkov); 59 (FRc) Sakmara distr., Mayorskoje vill., 1.VI.2008 (R.V. Filimonov); 3ㅇ. (DSc) Donskoye vill., Verblyuzhka Hill, Ural riv. flood-land, 23.V.2008 (S.V. Dedyukhin).

\section{Omiamima mollina (Boheman, 1834) (Omias)}

RECORDS. VL: CHV KIR UDM MRD UDM UR: BSH* [Jakovlev, 1910; Lugovaya, 1970; Egorov \& Isaev, 1998; Dedyukhin et al., 2005].

MATERIAL. Udmurtia: 1ex. (DSc), Malopurginsky distr., Yagan vill., Izh riv. flood land, 18.V.2003 (S.V. Dedyukhin), 1ex. (DSc), Alnashsky distr., Varzi-Yatshi vill., oak forest, 2.VI.2008 (S.V. Dedyukhin); Bashkortostan: $1+$ (FRc) Burzyansky distr., Shulgan-Tash Nature Reserve, 11.VI.2000 (P.V. Rudoiskatel).

Urometopus nemorum L. Arnoldi, 1965

RECORDS. VL: CHV SAM UDM ULY [Isaev, 1996; 2000 (moczarskii); Dedyukhin, 2010b; 2011c].

MATERIAL. Udmurtia: 1ex. (DSc), Troyeglazovo vill., Izh riv. flood land, oak forest, 15.VI. 2005 (S.V. Dedyukhin). 
tribe NASTINI (1 genus, 1 species)

Nastus (Nastus) goryi Boheman, 1842

= Nastus (Nastus) goryi sareptanus Faust, 1883.

RECORDS. VL: SAM UR: ORB* [Isaev, 1996].

MATERIAL. Orenburg Prov.: 3ex. (FRc) Gai distr., Kazachya Guberla vill., 18.V.2006 (R.V. Filimonov); $10^{7}$ (FRc) Orenburg, Zauralnaya Rosha, 28.IV.2007 (A.V. Rusakov).

tribe NAUPACTINI (1 genus, 1 species)

Mesagroicus poriventris Reitter, 1903

RECORDS. VL: ULY SAM UR: ORB* [Koblova, 1967; Isaev et al., 2004 (obscurus [MI] and poriventris)].

MATERIAL. Orenburg Prov.: 1 ㅇ (FRc) $20 \mathrm{~km} \mathrm{SSW} \mathrm{from}$ Orenburg, Donguz river valley, pitfall trap, 11-20.V.2007 (V.O. Kozminykh); $10^{\text {T }}$ (FRc) Akbulak distr., Orlovka vill., pitfall trap, 511.VII.2005 (V.A. Nemkov).

\section{tribe PSALLIDIINI (1 genus, 1 species)}

Psallidium maxillosum (Fabricius, 1792)

Curculio maxillosum Fabricius, 1792.

RECORDS. VL: SAM* ULY UR: ORB* [Isaev, 1994].

MATERIAL. Orenburg Prov.: 3ex. (FRc) $32 \mathrm{~km} \mathrm{E}$ from Orenburg, Priuralskij vill., 15.V.2007 (R.V. Filimonov); 1ex. (FRc) Sol'-Iletsk distr., $5 \mathrm{~km} \mathrm{~S}$ from Pervomaiskoje vill., Shubaragash forest, 18-21.V.2007 (R.V. Filimonov); 1ex. (FRc) Sol'-Iletsk distr., Pervomaiskoje vill. 2.V.2004 (unknown collector); 1ex. (DSc) Perevolotsky distr., Tshesnokovka vill., chalky steppe, 30.IV 2010 (S.V. Dedyukhin); 2ex. (DSc) Donskoye vill., Verblyuzhka Hill, stony steppe, sweeping at night, 29.VI. 2008 (S.V. Dedyukhin); Samara Prov.: 1ex. (DSc) Kinel'sky distr., Tshubovka vill., stony steppe, 2.VI.2010 (S.V. Dedyukhin); 1ex. (DSc) Stavropolsky distr., Zhiguli vill., oak forest, 9.V.2011 (S.V. Dedyukhin)

tribe POLYDRUSINI (2 genera, 15 species)

Polydrusus (Chlorodrosus) amoenus (Germar, 1824)

Melallites amoenus Germar, 1824

RECORDS. VL: KIR UDM UR: EKB* BSH* CHL KOM PER [Shernin, 1974; Tshaschina, 2002; Ermakov, 2003; Dedyukhin \& Tselischeva, 2011; Dedyukhin, 2010a; 2010b; 2011b]

MATERIAL. Bashkortostan: $70^{7} 4$ 오 (FRc) Mt. Maly Iremel, $1400 \mathrm{~m}$, on Aconogonon alpinum, 24.VII.1995 (R.V. Filimonov); Sverdlovsk Prov.: 1ex. (DSc) $10 \mathrm{~km} \mathrm{~W}$ of Revda, meadow in forest, 11.VIII.2010 (S.V. Dedyukhin).

Polydrusus (Eudipnus) mollis (Ström, 1768)

Curculio mollis Ström, 1768.

= Curculio micans (Fabricius, 1792).

RECORDS. VL: CHV KIR MRD SAM TAT UDM ULY UR: BSH* EKB ORB PER* [Lindeman, 1871 (micans); Lebedev, 1906; Jakovlev, 1910; Koblova, 1967; Roschinenko, 1972; Shernin, 1974 Isaev, 1994; 1996; Lagunov \& Novozhenov, 1996; Ermakov, 2003; Dedyukhin et al., 2005].

MATERIAL. Perm Reg.: 1ex. (DSc) Bardymsky distr., Sarash vill., on Populus tremula, 31.V.2011 (S.V.Dedyukhin); Bashkortostan: 1 ㅇ (FRc) Mt. Iremel, 8.VII.1999 (E.V. Guskova); Orenburg Prov.: 1 利 $\mathrm{NVc}$ ) Belyaevka distr., Orenburgsky N.R.: Burtinskaya Steppe, 4.VI.2003 (V.A. Nemkov); 6ㅇ (FRc) Kuvandyk distr., Maloye Churaevo vill., Shaitantau ridge, 10-11.VI.2009 (R.V. Filimonov); 1 + (FRc) idem, 23.V.2011 (R.V. Filimonov).

\section{Polydrusus (Eurodrusus) cervinus (Linnaeus, 1758)}

Curculio cervinus Linnaeus, 1758.

RECORDS. VL: CHV KIR MRD SAM TAT UDM ULY UR: CHL EKB ORB* PER [Lebedev, 1912; Koblova, 1967; Shernin, 1974; Isaev, 1994; 1996; Lagunov \& Novozhenov, 1996; Mikhailov, 1999; Dedyukhin et al., 2005; Dedyukhin, 2011b].

MATERIAL. Orenburg Prov.: $10^{7}$ (FRc) Sakmara distr., Mayorskoje vill., 1.VI.2008 (R.V. Filimonov); 1 ( (FRc) Tyulgan distr., Tashla vill., 29-31.V.2006 (V.E. Grigorjev).
Polydrusus (Eurodrusus) confluens Stephens, 1831

RECORDS. VL: CHV MRD TAT UDM ULY [Lindeman, 1871 (chrysomela); Csiki, 1901 (chrysomela); Isaev, 1994; Egorov \& Isaev, 1998; Dedyukhin et al., 2005; Dedyukhin, 2003; 2009].

MATERIAL. Tatarstan: $10^{\top} 1$ ( + (DSc) Yelabuga, Kama riv. bank, on Genista tinctoria, 28.VI.2011 (S.V. Dedyukhin).

Polydrusus (Eurodrusus) pilosus Gredler, 1866

RECORDS. VL: CHV KIR MRD SAM TAT UDM* UR: CHL EKB ORB PER [Lebedev, 1906; Jakovlev, 1910; Koblova, 1967; Shernin, 1974; Isaev, 1994; 1996; Lagunov \& Novozhenov, 1996; Mikhailov, 1999; Ermakov, 2003; Database of IEPA, 2009].

MATERIAL. Udmurtia: 1ex. (DSc) Votkinsky distr., Ledukhi vill., boreal forest, on Sorbus aucuparia, 23.V.1996 (S.V. Dedyukhin), $10^{\top} 3$ ( (DSc) Yakshur-Bod'insky distr., Selytshka vill., boreal forest, on Alnus incana and Betula pubescens, 16.V.2002 (S.V. Dedyukhin).

Polydrusus (Eustolus) corruscus Germar, 1824

RECORDS. VL: CHV KIR MRD SAM TAT UDM ULY UR: CHL* ORB* PER [Lebedev, 1906; 1912; Jakovlev, 1910; Shernin, 1974; Isaev, 1994; 1996; Egorov \& Isaev, 1998; Dedyukhin et al., 2005].

MATERIAL. Kirov Prov.: 4 ex., (DSc) Kilmezsky distr., Tautovo vill., Kil'mez' riv. bank, on Salix acutifolia and S. viminalis, 3.VII.2011 (S.V. Dedyukhin); Chelyabinsk Prov.: $20^{7} 3$ 우 (FRc) Kartaly distr., Varshavka vill., on Salix viminalis, 15-16.VII.1998 (R.V. Filimonov); Orenburg Prov.: $4 \bigcirc^{7} 8$ ㅇ (FRc) Sol'-Iletsk distr., Novoiletsk vill., 25-27.V.2008 (R.V. Filimonov).

Polydrusus (Eustolus) flavipes (De Geer, 1775)

Curculio flavipes De Geer, 1775

RECORDS. VL: CHV KIR MRD SAM TAT UDM ULY UR: CHL EKB ORB* PER [Lindeman, 1871; Lebedev, 1906; 1912; Jakovlev, 1910; Shernin, 1974; Isaev, 1994; Lagunov \& Novozhenov, 1996; Egorov \& Isaev, 1998; Mikhailov, 1999; Dedyukhin et al., 2005; Database of IEPA, 2009; Dedyukhin, 2009; 2010d; Dedyukhin \& Tselischeva, 2011].

Polydrusus (Eustolus) impressifrons Gyllenhal, 1834 RECORDS. VL: MRD CHV TAT UR: PER [Lindeman, 1871 (flavovirens); Lebedev, 1906; Egorov \& Isaev, 2006]. NOTE. All records are to be confirmed.

Polydrusus (Eustolus) pterygomalis Boheman, 1840

RECORDS. VL: CHV MRD SAM TAT UDM ULY UR: CHL EKB [Lebedev, 1906; Lagunov \& Isaev, 1996; 2000; Novozhenov, 1996; Dedyukhin et al., 2005; Dedyukhin, 2009; 2010d].

Polydrusus (Polydrusus) fulvicornis (Fabricius, 1792)

Curculio fulvicornis Fabricius, 1792.

$=$ Curculio ruficornis (Bonsdorff, 1785) [HN].

RECORDS. VL: CHV KIR MAR* MRD TAT UDM ULY UR: BSH CHL EKB KOM ORB PER [Lebedev, 1906; Jakovlev, 1910; Shernin, 1974; 1912; Egorov \& Isaev, 1998; Ermakov, 2003; Dedyukhin et al., 2005; Isaev, 2000].

MATERIAL. Mari El: $10^{\prime} 2+$ (KUMN) $61 \mathrm{~km}$ SSE Yoshkar-Ola, Mari Chodyra N.P., Ilet' riv. valley, N 56 $6^{\circ}$ '4.51", E 48²5'59.45", flood-land, beating, 19.VII.2006 (N.N. Yunakov); Bashkortostan: 1 ' (FRc) Mt. Iremel, 8.VII.1999 (E.V. Guskova); Chelyabinsk Prov.: $10^{7}$ (FRc) Zlatoust distr., Urzhumka railway station, Mt. Alexandrovskaya Sopka, 7.VIII.1996 (R.V. Filimonov); 10ㄱ, 1 ( (FRc) Miass city distr., Turgoyak railway station, on Betula sp., 3.VII.2000 (R.V. Filimonov); 10, 1 ㅇ (FRc) idem, 11.VII.2000 (R.V. Filimonov).

\section{Curculio tereticollis (De Geer, 1775)}

Curculio tereticollis De Geer, 1775.

$=$ Curculio undatus Fabricius, 1781 .

RECORDS. VL: CHV KIR MAR* MRD SAM TAT UDM ULY UR: BSH* CHL EKB KOM ORB* PER [Lindeman, 1871; Lebedev, 1906; 1912; Jakovlev, 1910; Koblova, 1967; Shernin, 1974; Isaev, 1994; 1996; Lagunov \& Novozhenov, 1996; Mikhailov, 
1999; Ermakov, 2003; Dedyukhin, 2003; Dedyukhin et al., 2005; Database of IEPA, 2009; Dedyukhin \& Tselischeva, 2011].

MATERIAL. Mari El: $1+$ (KUMN) 61 km SSE Yoshkar-Ola, Mari Chodyra N.P., Ilet' riv. valley, N 569'4.51", E 48²5'59.45", flood-land, beating, 19.VII.2006 (N.N. Yunakov); Bashkortostan: $2 \sigma^{7} 1$ ㅇ (FRc) Mt. Iremel, 8.VII.1999 (E.V. Guskova); $2 \sigma^{7} 4$ + (FRc) idem, 1000-1300 m, on Betula sp., 1-4.VI.2000 (R.V. Filimonov); Orenburg Prov.: 1ex. (RAc) $14 \mathrm{~km}$ NE from Orenburg, Grebeni vill., 14.VI.2006 (A.V. Rusakov); 1ex. (NVc) Novosergievka distr., Staraya Belogorka vill., 14.VI.2008 (V.A. Nemkov); 1ex. (NVc) Tashla distr., Trudovoye vill., 28.IV-2.V.2008 (V.A. Nemkov).

Polydrusus (Polydrusus) picus (Fabricius, 1792)

Curculio picus Fabricius, 1792.

RECORDS. VL: SAM ULY [Isaev, 1994; 1996; 2007].

Polydrusus (Orodrusus) obliquatus Faust, 1884 RECORDS. UR: ORB*

MATERIAL. Orenburg Prov.: 19 (FRc) 14 km NE from Orenburg, Grebeni vill., 1.VI.2004 (A.M. Shapovalov); 19 (ZIN) SolIletsk distr., Izobilnoye vill., 9.VI.2004 (V.A. Nemkov).

NOTE. This is a first record of Orodrusus in Russia and

Europe. It is parthenogenetic species occurring in Middle Asia, Kazakhstan, Iran, and Afghanistan.

\section{Polydrusus (Scythodrusus) inustus Germar, 1824}

RECORDS. VL: CHV KIR MRD SAM TAT UDM ULY UR: BSH CHL* EKB ORB* [Lebedev, 1925(pilifer); Jakovlev, 1910; Isaev, 1994; 1996; 2007 (pilifer); Dedyukhin et al., 2005; Sapronov, 2008; Dedyukhin, 2009].

MATERIAL. Chelyabinsk Prov.: $1+$ (FRc) Chelyabinsk, Fedorovka suburb, on Sorbus aucuparia, 17-18.VI.2005 (R.V. Filimonov); 10 + (FRc) idem, on Aronia mitschurinii, Prunus domestica, Rubacer odoratum, 10-16.VI.2007 (R.V. Filimonov); Orenburg Prov.: 3 + (NVc) Orenburg, on Malus sp., 1.VI.1988 (V.A. Nemkov); 1 ( $(\mathrm{NVc})$ 1 우 (FRc) Orenburg, Rostoshi suburb, 30.V.2007 (V.A. Nemkov).

\section{Liophloeus (Liophloeus) tessulatus (Müller, 1776)}

Curculio tessulatus Müller, 1776.

RECORDS. VL: CHV KIR MRD SAM TAT UDM ULY UR: BSH* CHL* ORB* PER* [Lebedev, 1906; Jakovlev, 1910; Shernin, 1974; Isaev, 1994; 1996; Egorov \& Isaev, 1998; Dedyukhin et al., 2005].

MATERIAL. Bashkortostan: $1+$ (FRc) Burzyansky distr., Shulgan-Tash Nature Reserve, 15.VI.2000 (P.V. Rudoiskatel); Orenburg Prov.: 2 (FRc) $14 \mathrm{~km}$ NE from Orenburg, Grebeni vill., 24.V.2006 (R.V. Filimonov); 1 ㅇ (FRc) Tyulgan distr., Tashla vill., 6-7.VII.2009 (A.M. Shapovalov); Chelyabinsk Prov.: 19 (FRc) 10 km SSW from Chelyabinsk, Sargazy vill., 12.VII.1990 (R.V. Filimonov); 1 오 (FRc) $10 \mathrm{~km} \mathrm{~S}$ from Chelyabinsk, Voznesenka vill., 13.VI.2008 (R.V. Filimonov); Perm' Reg.: 1 \% (DSc) Bardymskyi distr., Sarashi vill., Tulva riv. bank, 31.V. 2011 (S.V. Dedyukhin)

tribe SCIAPHILINI (10 genera, 11 species)

Archeophloeus inermis (Boheman, 1843)

Trachyphloeus inermis Boheman, 1843.

= Archeophloeus hypocrita Iablokov-Khnzorian, 1959 [synonymy after Borovec, 2009].

RECORDS. VL: ULY UR: CHL* ORB* [Isaev, 1994].

MATERIAL. Chelyabinsk Prov.: 21ex. (FRc) Bredy distr., Naslednitskij vill., 15-18.V.2008 (R.V. Filimonov); Orenburg Prov.: 10 ex. (FRc) $20 \mathrm{~km} \mathrm{SSW}$ from Orenburg, Donguz river valley, in mat of moss, 8-17.V.2007 (R.V. Filimonov); 2ex. (FRc) idem, pitfall trap, 11-20.V.2007 (V.O. Kozminykh); 1 ex. (FRc) 14 km NE from Orenburg, Grebeni vill., 13.V.2007 (R.V. Filimonov).

\section{Foucartia squamulata (Herbst, 1795)}

Curculio squamulata Herbst, 1795.

RECORDS. VL: CHV KIR MRD SAM TAT UDM ULY UR: BSH* CHL ORB PER [Lebedev, 1906; Jakovlev, 1910; Koblova, 1967; Isaev, 1994; 1996; Mikhailov, 1999; Dedyukhin, 2003; 2009; 2010a; 2010b; 2011b; Dedyukhin et al., 2005].
MATERIAL. Baschkortostan: 3ex. (DSc) Sterlitamak, Shikhan Tratau hill, 31.V.2010 (S.V. Dedyukhin); Orenburg Prov.: 1ex. (NVc) Novosergievka distr., Staraya Belogorka vill., 14.VI.2008 (V.A. Nemkov); 1ex. (NVc) Tashla distr., Trudovoye vill., 13.VI.2007 (V.A. Nemkov); 1ex. (NVc) idem, 12.VI.2008 (V.A. Nemkov); 2ex. (NVc) Kuvandyk distr., Krasnosshyokovo vill., 26.VI.2000 (V.A. Nemkov).

Sciaphobus rubi (Gyllenhal, 1813)

Curculio rubi Gyllenhal, 1813.

= Thylacites ningnidus (Germar, 1824).

RECORDS. VL: CHV KIR MRD SAM TAT UDM ULY UR: BSH* CHL ORB* PER [Lindeman, 1871 (ninguidus) [MS]; Lebedev, 1906; Jakovlev, 1910; Isaev, 1994; 1996; Mikhailov, 1999; Dedyukhin, 2009; 2011b].

MATERIAL. Bashkortostan: $1+$ (FRc) Burzyansky distr., Akbulatovo-Mulatovo vill., 5.VI.2000 (P.V. Rudoiskatel); 19 (FRc) Burzyansky distr., Shulgan-Tash Nature Reserve, 6.VI.2000 (P.V. Rudoiskatel); Chelyabinsk Prov.: 19 (FRc) Chesma distr., Chernoborskij vill., 22-25.VI.2011 (R.V. Filimonov); 2 (FRc) idem, on Rubus idaeus L., 29.VI-2.VII.2011 (R.V. Filimonov); Orenburg Prov.: 2 ( (NVc, FRc) Tashla distr., Trudovoye vill., 12.VI.2008 (V.A. Nemkov); 19 (FRc) Kuvandyk distr., Maloye Churaevo vill., Shaitantau ridge, 10.VI.2009 (R.V. Filimonov).

Sciaphilus asperatus (Bonsdorff, 1785)

Curculio asperatus Bonsdorff, 1785.

= Curculio muricatus (Fabricius, 1792).

RECORDS. VL: CHV KIR MAR* MRD SAM TAT UDM ULY UR: BSH CHL EKB PER* [Lindeman, 1871 (muricatus); Lebedev, 1906; Jakovlev, 1910; Isaev, 1994; 1996; Lagunov \& Novozhenov, 1996; Dedyukhin et al., 2005; Database of IEPA, 2009; Sapronov, 2010b].

MATERIAL. Mari El: 4ㅇ (ZMUN) Yoshkar-Ola, right bank of Malaya Kokshaga riv., 100 m, N56 39' 12.1' E47 56' 07.7', leaf litter in Quercus-Abies forest, 13.VIII.2008 (N.N. Yunakov); Permsky Reg.: 1 ㅇ (DSc) Kungur, Spasskaya Mt., 16.VII.2009 (S.V. Dedyukhin); Bashkortostan: 1 ( (FRc) Burzyansky distr., ShulganTash Nature Reserve, 17.VI.2000 (P.V. Rudoiskatel).

\section{Barypeithes (Exomias) lebedevi Roubal, 1926}

RECORDS. VL: CHV KIR MRD TAT ULY UDM [Lebedev, 1925 (Omias ?nitidus); Roubal, 1926; Isaev, 2000 (Omiamima concinna); Egorov \& Isaev, 2001; Yuferev, 2001a (Omiamima nitida); 2003, 2004; Isaev et al., 2004; Dmitrieva, 2005; Dedyukhin et al., 2005; Dedyukhin, 2010b; 2011c; Dedyukhin, Tselischeva, 2011].

Barypeithes (Exomias) pellucidus Boheman, 1834

RECORDS. VL: CHV UDM ULY [Isaev, 1994; Egorov \& Isaev, 1998; Dedyukhin, 2010b; 2010d].

\section{Eusomus ovulum Germar, 1824}

= Eusomus beckeri Tournier, 1874.

RECORDS. VL: CHV KIR MAR* MRD SAM TAT UDM ULY UR: BSH* CHL EKB ORB PER [Lindeman, 1871; Lebedev, 1906; Arnoldi, 1954 (beckeri); Koblova, 1967 (beckeri); Esyunin \& Kozminykh, 1992 (beckeri); Isaev, 1994; 1996; Egorov \& Isaev, 1998; Mikhailov, 1999; Yuferev, 2001a; 2001b; Tshaschina, 2002; Dedyukhin, 2003; 2011b; Dedyukhin et al., 2005; Tshitshikov \& Legalov, 2008; Database of IEPA, 2009].

MATERIAL. Mari El: 4 오 (ZIN) Yoshkar-Ola, flood-lands of Malaya Kokshaga riv., meadow near oak forest 18.VII.2006 (N.N. Yunakov); Bashkortostan: 5ex. (DSc) Sterlitamak, Shikhan Tratau hill, steppe, 31.V.2010 (S.V. Dedyukhin); Orenburg Prov.: 2ex. (NVc) Tashla distr., Trudovoye vill., 21-26.VI.2002 (V.A. Nemkov); 1ex. (NVc) Belyaevka distr., Orenburgsky N.R.: Burtinskaya Steppe, 5-10.VII.2002 (V.A. Nemkov).

\section{Euidosomus acuminatus (Boheman, 1840)}

Eusomus acuminatus Boheman, 1840.

RECORDS. VL: CHV SAM ULY UR: BSH* CHL ORB [Koblova, 1967; Isaev, 1994; Egorov \& Isaev, 1998; Mikhailov, 1999]. 
MATERIAL. Baschkortostan: 3ex. (DSc), Sterlitamak, Shikhan Tratau hill, stony steppe, 30.V.2010, (S.V. Dedyukhin).

Eusomatus virens (Boheman, 1833)

Eusomus virens Boheman, 1833.

RECORDS. UR: ORB*

MATERIAL. Orenburg Prov.: $10^{7}$ (FRc) Sol-Iletsk distr., Shibyndy Ravine, chalky hills, 25.V.1996 (A.V. Rusakov); 20 (FRc), idem, 4-6.VI.2004 (A.M. Shapovalov); 40 2 ㅇ (FRc) SolIletsk distr., Troitsk vill., steppe on chalky hills, 8-22.VI.2008 (V.A. Nemkov).

\section{Brachysomus (Brachysomus) echinatus (Bonsdorff, 1785)}

Curculio echinatus Bonsdorff, 1785.

RECORDS. VL: CHV KIR MAR* MRD SAM TAT UDM ULY UR: BSH CHL* EKB KOM* ORB* PER* [Lindeman, 1871 (Platytarsus echinatus); Lebedev, 1906; Jakovlev, 1910; Isaev, 1994; 1996; Egorov \& Isaev, 1998; Dedyukhin et al., 2005; Database of IEPA, 2009; Dedyukhin \& Tselischeva, 2011]

MATERIAL. Komi Autonomous Republic:1 1 (ZIN) Povelitsa, 11-20.VI.1976 (A.L. Lobanov); Mari El: 4우 (ZMUN) Yoshkar-Ola, right bank of Malaya Kokshaga riv., $100 \mathrm{~m}$, N56 39 12.1' 'E47 56' 07.7', , leaf litter in Quercus-Abies forest, 13.VIII.2008 (N.N. Yunakov); Bashkortostan: 1 (아 (FRc) Burzyansky distr., Akbulatovo-Mulatovo vill., 8.VI.2000 (P.V. Rudoiskatel); 1 ㅇ (FRc) Burzyansky distr., Shulgan-Tash Nature Reserve, 11.VI.2000 (P.V. Rudoiskatel); Chelyabinsk Prov.: 1 오 (FRc), Miass city distr., Syrostan vill., 10.VI.1999 (R.V. Filimonov); 19 (FRc), idem, 15.VII.1999 (R.V. Filimonov); 5 ㅇ (FRc), Miass city distr., Turgoyak railway station, pitfaill traps, 3-22.VII.2000 (R.V. Filimonov); 2 (FRc) $10 \mathrm{~km} \mathrm{SSW}$ from Chelyabinsk, Sargazy vill., 15.V.1997 (R.V. Filimonov); Orenburg Prov.: 1ex. (NVc) Tashla distr., Trudovoye vill., 21-26.VI.2002 (V.A. Nemkov); 2 ㅇ (FRc) $14 \mathrm{~km} \mathrm{NE}$ from Orenburg, Grebeni vill., 28.V.2009 (R.V. Filimonov); Perm' Reg.: Dobryansky distr., Porlazna, 2.VI.2010 (S.V. Dedyukhin)

Amicromias mimicus Yunakov, 2005

RECORDS. VL: SAM [Yunakov, 2005].

MATERIAL. 2 9 (ZIN) Russia, Samara Prov., Sinii Syrt, 13 km SSW Kostino vill., 7-16.VII.1993 (Smelyansky); 107, holotype (ZIN) idem, 10-21.VI.1994 (Smelyansky).

NOTE. This species only from type locality is known.

tribe BRACHYDERINI (4 genera, 4 species)

Brachyderes incanus (Linnaeus, 1758)

Curculio incanus Linnaeus, 1758.

RECORDS. VL: MAR* MRD SAM TAT UDM ULY [Lebedev, 1906; Yuferev, 2004; Dedyukhin et al., 2005; Roschinenko, 1981; Isaev, 1994; Dedyukhin, 2003].

MATERIAL. Mari El: $5 \sigma^{\top} 3+$ (ZIN) Gornomariisky distr., 90 $\mathrm{km}$ WSW of Yoshkar-Ola, $16 \mathrm{~km}$ NE of Yurkino vill., nr Karasjar Lake, 102 m, N56³6' 42.8' 'E46²3' 37.5'” 28.VII.2005 N. Yunak-

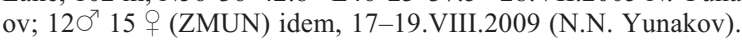

Pholicodes inauratus arzanovi Davidian, 1992

RECORDS. UR: ORB*.

MATERIAL. Orenburg Prov.: 2ex. (NVc), 1ex. (FRc) Tashla distr., Trudovoye vill., 12.VI.2006 (V.A. Nemkov).

Strophosoma capitatum (De Geer, 1775)

Curculio capitatum De Geer, 1775.

= Curculio obesus Marsham, 1802 [HN].

$=$ Curculio coryli Gyllenhal, 1813 [MI].

RECORDS. VL: CHV KIR MAR MRD SAM TAT UDM ULY UR: BSH CHL EKB KOM ORB PER [Lindeman, 1871 (coryli, obesus); Csiki, 1901 (obesus); Lebedev, 1906; Jakovlev, 1910; Boitsova, 1931; Shiperovitsh, 1939; Polozhentsev, 1947 (coryli) Shernin, 1974; Roschinenko, 1981; Isaev, 1994; 1996; Lagunov \& Novozhenov, 1996; Dedyukhin et al., 2005; Matveev, 2007; Dedyukhin, Tselischeva, 2011].
Pelletierius albosignatus (Boheman, 1840)

Strophosomus albosignatus Boheman, 1840.

= Strophosomus albolineatus Seidlitz, 1867

RECORDS. VL: CHV MRD TAT ULY UR: ORB [Lebedev, 1912; Arnoldi, 1954 (Strophosomus albolineatus); Lugovaya, 1970; Isaev, 1994; Dmitrieva, 2005].

MATERIAL. Orenburg Prov.: $10^{7}$ (FRc) Buzuluk distr., Buzulukskij Bor N.P., 12.V.2007 (K.A. Khristina); 1 (FRc) Sol'-Iletsk distr., $5 \mathrm{~km} \mathrm{~S}$ from Pervomaiskoje vill., Khobda riv., 18.V.2006 (K.A. Khristina); 29 (FRc) idem, 18-21.V.2007 (R.V. Filimonov); 4ex. (DSc) Akbulaksky distr., Akbulak, Ilek riv. flood-land, 2.V.2010 (S.V. Dedyukhin).

tribe CNEORHININI (2 genera, 2 species)

Attactagenus albinus (Boheman, 1833)

Cneorhinus albinus Boheman, 1833.

RECORDS. VL: CHV MRD ULY [Egorov \& Isaev, 1998; Isaev, 2000; Dmitrieva, 2005].

Philopedon plagiatum (Schaller, 1783)

Curculio plagiatum Schaller, 1783.

RECORDS. VL: MRD [Rutshin, 2009].

tribe TANYMECINI (5 genera, 10 species)

Tanymecus palliatus (Fabricius, 1787)

Curculio palliatus Fabricius, 1787.

RECORDS. VL: CHV KIR MAR* MRD SAM TAT UDM ULY

UR: CHL EKB KOM ORB PER* [Lindeman, 1871; Csiki, 1901; Lebedev, 1906; Jakovlev, 1910; Bei-Bienko, 1946; Koblova, 1967; Roschinenko, 1972; Shernin, 1974; Isaev, 1994; Lagunov \& Novozhenov, 1996; Mikhailov, 1999; Dedyukhin et al., 2005; Tshitshikov \& Legalov, 2008; Database of IEPA, 2009; Dedyukhin \& Tselischeva, 2011].

MATERIAL. Mari El: 2 ( $\mathrm{ZIN})$ Medvedevo distr., Bolshaya Kokshaga N.R., nr Shaptunga vill., 150 m, N56³8' 31.92'” E47¹3' 45.41" meadow, sweeping, 29.vii.2006 (N.N. Yunakov); Parm Reg.: 2 ex (DSc) Kungur, Spasskaya Mt., 14.VII.2009 (S.V. Dedyukhin); $10^{7} 1$ (으 (ZIN) "Perm"; Orenburg Prov.: 19 (FRc) Sakmara distr., Mayorskoje vill., 24.V.2007 (R.V. Filimonov); 1ㅇ (FRc) idem, 1.VI.2008 (R.V. Filimonov); $10^{\top} 3$ 9 (FRc) Svetly distr., 14 km NNW from Svetly town, Obalykol' lake, 6-7.VI.2006 (R.V. Filimonov).

Megamecus argentatus (Gyllenhal, 1840)

Tanymecus argentatus Gyllenhal, 1840.

RECORDS. VL: ULY UR: CHL* ORB [Isaev, 1994; Nemkov \& Rusakov, 1995].

MATERIAL. Orenburg Prov.: 5ex. (FRc) Svetly distr., 14 km NNW from Svetly town, Obalykol' lake, 6-7.VI.2006 (R.V. Filimonov); Chelyabinsk Prov.: 1 (FRc) Chesma distr., Chernoborskij vill., on Rumex confertus, 29.VI.-2.VII.2011 (R.V. Filimonov)

Megamecus variegatus (Gebler, 1830)

Tanymecus variegatus Gebler, 1830.

RECORDS. UR: ORB*.

MATERIAL. Orenburg Prov.: 1ex. (NVc) Sol'-Iletsk distr., Novoiletsk vill., 20-22.V.2003 (A.M. Shapovalov).

Chlorophanus viridis (Linnaeus, 1758)

Curculio viridis Linnaeus, 1758.

RECORDS. VL: CHV KIR MAR* MRD TAT UDM ULY UR: BSH CHL KOM ORB PER [Lindeman, 1871; Csiki, 1901; Lebedev, 1906; Jakovlev, 1910; Roschinenko, 1972; 1981; Shernin, 1974; Isaev, 1994; Esyunin \& Kozminykh, 1992; Ismailova, 1993; Yuferev, 2001 (graminicola) [MI]; Dedyukhin et al., 2005; Database of IEPA, 2009; Dedyukhin \& Tselischeva, 2011].

MATERIAL. Mari El: 19 (KUMN) 61 km SSE Yoshkar-Ola, Mari Chodyra N.P., Ilet' riv. valley, N 569'4.51", E 48²5'59.45", flood-land, beating, 19.VII.2006 (N.N. Yunakov); Chelyabinsk Prov.: 1 을 (FRc) Verkhneuralsk distr., Maly Kizil riv., 18-25.VI.2005 (N.A. Rezchikova). 
Chlorophanus sellatus (Fabricius, 1798)

Curculio sellatus Fabricius, 1798

RECORDS. VL: CHV KIR SAM TAT UDM ULY UR: BSH CHL ORB [Lebedev, 1906; Roschinenko, 1981 (graminicola) [MI]; Ismailova, 1993; Isaev, 1994; 1996; Egorov \& Isaev, 1998; Dedyukhin, 2010b; 2011a].

MATERIAL. Kirov Prov.: 3ex. (DSc) Kil'mezsky distr., Tautovo vill., Kil'mez' riv. flood land, 17.VI.1970 (V.I. Roschinenko) Orenburg Prov.: 23ex. (FRc) Sol'-Iletsk distr., Novoiletsk vill., on Salix sp., 25-27.V.2008 (R.V. Filimonov \& A.M. Shapovalov).

Chlorophanus graminicola (Olivier, 1807)

Curculio graminicola Olivier, 1807.

RECORDS. VL: CHV MRD TAT ULY UR: ORB [Lebedev, 1906; Ismailova, 1993].

MATERIAL. Orenburg Prov.: $50^{7} 9$ 우 (FRc) Sol'-Iletsk distr., Novoiletsk vill., on Salix sp., 25-27.V.2008 (R.V. Filimonov \& A.M. Shapovalov).

Chlorophanus caudatus Fåhraeus, 1840

RECORDS. UR: ORB [Ismailova, 1993].

MATERIAL. Orenburg Prov.: $10^{7} 19$ (FRc) Ilek distr., Nizhneoziornoye vill, 8.VIII.2006 (A.V. Rusakov); 12ex. (FRc) Sol'Iletsk distr., Novoiletsk vill., on Salix sp., 25-27.V.2008 (R.V. Filimonov \& A.M. Shapovalov).

\section{Phacephorus nebulosus Fåhraeus, 1840}

Tanymecus nebulosus Fåhraeus, 1840.

RECORDS. UR: ORB*

MATERIAL. MATERIAL. Orenburg Prov: 19 (FRc) Svetly distr., Shalkar-Ega-Kara lake, 26.IV.2001 (A.V. Rusakov); $20^{7} 39$ (FRc) Svetly distr., $14 \mathrm{~km}$ NNW from Svetly town, Obalykol' lake, 6-7.VI.2006 (R.V. Filimonov).

Phacephorus hirtellus Faust, 1885

RECORDS. UR: ORB*

MATERIAL. Orenburg Prov.: $10^{7}$ (DSc) 1 오 (ZIN) Akbulak distr., $7 \mathrm{~km} \mathrm{~S}$ of Fedorovka vill., Bazar-Bai Mt., stony steppe, under stones, 2.V.2010 (S.V. Dedyukhin).

NOTE. This is a first record in Russian and European fauna.

Cycloderes pilosulus (Herbst, 1795)

Curculio pilosulus Herbst, 1795.

$=$ Curculio pilosus (Fabricius, 1792) [HN].

RECORDS. VL: CHV MRD SAM TAT ULY UR: CHL ORB* PER [Lindeman, 1871 (Thylacites pilosus); Lebedev, 1906; Jakovlev, 1910; Arnoldi, 1954 (Thylacites pilosus); Koblova, 1967; Lugovaya, 1970; Shernin, 1974; Esyunin \& Kozminykh, 1992; Isaev, 1994; 1996; Mikhailov, 1999; Dedyukhin, 2010a; 2010b; 2011b].

MATERIAL. Orenburg Prov.: 39 (FRc) Sakmara distr., Mayorskoje vill., 24.V.2007 (R.V. Filimonov); 1 우 (DSc) Belyaevsky distr., Donskoye vill., Ural riv. Flood land, dry meadow, 25.V.2008 (S.V. Dedyukhin); 3ex. (NVc) Sol-Iletsk distr., Troitsk vill., steppe on chalky hills, 8-22.VI.2008 (V.A. Nemkov); 1 ㅇ (FRc) Svetly distr., $14 \mathrm{~km} \mathrm{NNW}$ from Svetly town, Obalykol' lake, 6.VI.2006 (R.V. Filimonov).

\section{tribe SITONINI (2 genera, 20 species)}

Charagmus griseus (Fabricius, 1775)

Curculio griseus Fabricius, 1775.

RECORDS. VL: CHV MAR* MRD [Lugovaya, 1970; Egoroy \& Isaev, 1998; 2006; Isaev et al., 2004]

MATERIAL. Mari El: 2ex. (ZIN) Zvenigovo distr., floodplain of the Ilet' river, alder forest, 1-14.VI.1988 (V.A. Matveev).

Sitona ambiguus Gyllenhal, 1834

RECORDS. VL: CHV KIR MAR* MRD SAM UDM ULY UR BSH CHL EKB KOM ORB PER [Isaev, 1994; 1996; Lagunov \& Novozhenov, 1996; Yuferev, 2001a; Sapronov, 2010b; Dedyukhin, 2011b; Dedyukhin \& Tselischeva, 2011].
MATERIAL. Mari El: $20^{2} 4$ 우 (ZIN) Gornomariisky distr., 90 $\mathrm{km}$ WSW of Yoshkar-Ola, $16 \mathrm{~km}$ NE of Yurkino vill., nr Karasjar Lake, 102 m, N56 $36^{\prime}$ 42.8' E46²3' 37.5', 28.VII.2005 (N.N. Yunakov); $10^{7} 4$ 오 (ZIN) Medvedevo distr., Bolshaya Kokshaga N.R., nr Shaptunga vill., $150 \mathrm{~m}$, N56 $38^{\circ}$ 31.92' E47¹3' 45.41', meadow, sweeping, 29.VII.2006 (N.N. Yunakov).

\section{Sitona callosus Gyllenhal, 1834}

RECORDS, VL: CHV MRD SAM ULY UR: CHL ORB* [Lugovaya, 1970; Isaev, 1994; 1996; Lagunov \& Novozhenov, 1996; Egorov \& Isaev, 1998; Mikhailov, 1999; Tshitshikov \& Legalov, 2008].

MATERIAL. Orenburg Prov.: $10^{\top}$ (FRc) Sakmara distr., Mayorskoye vill., 24.V.2007 (R.V. Filimonov); 2 (FRc) idem, 1.VI.2008 (R.V. Filimonov); $10^{7}$ (FRc), 1ex. (NVc) Belyaevka distr., Orenburgsky N.R.: Burtinskaya Steppe, 9.VII.2006 (V.A. Nemkov).

Sitona cylindricollis Fåhraeus, 1840

RECORDS. VL: CHV KIR MAR* MRD SAM TAT* UDM ULY UR: BSH CHL EKB ORB* PER* [Koblova, 1967; Isaev, 1994; 1996; Lagunov \& Novozhenov, 1996; Mikhailov, 1999; Yuferev, 2001a; 2001b; Dedyukhin et al., 2005; Tshitshikov \& Legalov, 2008; Database of IEPA, 2009 (cylindricus) [MS]; Sapronov, 2010b].

MATERIAL. Mari El: 1 우 (ZIN) Yoshkar-Ola, right bank of Malaya Kokshaga riv., 100 m, N56 39' 12.1'" E47 56' 07.7' meadow near Quercus-Abies forest, 10.VIII.2009 (N.N. Yunakov); Perm' Reg.: 2 (ZIN) "Perm"; 1 ex. (DSc) Kungur distr., Gorbunyata vill., carbonate rocks, 29.VI.2007 (S.V. Dedyukhin); 6ex. (DSc) Nytvensky distr., Dybki vill., on Melilotus albus, 28.VI. 2010 (S.V. Dedyukhin); Tatarstan: 10" (ZIN) "Kazan"; 1ex. (DSc) Elabuga, Kama bank, on Melilotus albus, 26.VI. 2010 (S.V. Dedyukhin); Bashkortostan: 1 우 (FRc) Mt. Iremel, 1000-1300m, 1-4.VI.2000 (R.V. Filimonov); Orenburg Prov.: 1ex. (NVc) Kuvandyk distr., Aituar vill., Orenburgsky N.R.: Aituarskaya Steppe, 5-10.VII.2002 (V.A. Nemkov); 1 오 (FRc) Buzuluk distr., Buzulukskij Bor N.P., 12.V.2007 (K.A. Khristina); 4ex. (RAc) $20 \mathrm{~km} \mathrm{SSW} \mathrm{from} \mathrm{Oren-}$ burg, Donguz river valley, 18.V.2005 (K.A. Khristina)

\section{Sitona hispidulus (Fabricius, 1776)}

Curculio hispidulus Fabricius, 1776

= Sitona foedus Gyllenhal, 1834

RECORDS. VL: CHV KIR MAR* MRD SAM TAT UDM ULY UR: BSH* CHL KOM ORB PER [Bei-Bienko, 1946; Ryazantsev et al., 1966; Lagunov \& Novozhenov, 1996; Isaev; 1994; 1996; Yuferev, 2001a; Dedyukhin et al., 2005; Dedyukhin, 2010d; Dedyukhin \& Tselischeva, 2011].

MATERIAL. Mari El: $10^{7} 1$ ( Malaya Kokshaga riv., 100 m, N56³9' 12.1' 'E4756' 07.7' ' meadow near Quercus-Abies forest, 10.VIII.2009 (N.N. Yunakov); Bashkortostan: 3ex. (ZIN) Belebey, 10.VII.1907 (Grigorjev)

Sitona humeralis Stephens, 1831

RECORDS, VL: MAR* MRD TAT ULY UR: BSH CHL ORB* [Lebedev, 1925; Esyunin \& Kozminykh, 1992; Isaev, 1994; 2007$].$

MATERIAL. Mari El: $3 \sigma^{\top} 1+($ ZIN) Yoshkar-Ola, right bank of Malaya Kokshaga riv., 100 m, N56³9' 12.1' 'E4756' 07.7' ' meadow near Quercus-Abies forest, 10.VIII.2009 (N.N. Yunakov); Orenburg Prov.: 1ex. (RAc) $30 \mathrm{~km} \mathrm{NE}$ from Saraktash town, 3.VII.2003 (A.V. Rusakov).

Sitona inops Gyllenhal, 1832

RECORDS. VL: CHV KIR MRD SAM TAT UDM ULY UR: CHL EKB ORB* PER* [Lebedev, 1906; Koblova, 1967; Esyunin \& Kozminykh, 1992; Isaev, 1994; 1996; Yuferev, 2001a; Dedyukhin et al., 2005; Dedyukhin, 2010b].

MATERIAL. Perm' Reg.: 4ex. (DSc) Dobryansky distr. Lunezhki vill., Kama riv. bank, on Medicago sativa, 2.VI.2010 (S.V. Dedyukhin); Orenburg Prov.: 1ex. (RAc) $20 \mathrm{~km} \mathrm{SSW} \mathrm{from} \mathrm{Orenburg,} \mathrm{Donguz} \mathrm{riv.}$ valley, 18.V.2005 (K.A. Khristina); 1 ex. (NVc) Belyaevka distr., Orenburgsky N.R.: Burtinskaya Steppe, 5.VII.2008 (V.A. Nemkov); 1ex. (NVc) Kuvandyk distr., Aituar vill., Orenburgsky N.R.: Aituarskaya Steppe, Shinbutak Ravine, 31.V.2007 (V.A. Nemkov). 
Sitona languidus Gyllenhal, 1834

RECORDS. VL: KIR MRD SAM TAT UDM ULY UR: BSH* ORB* [Isaev, 1990; 1994; Dedyukhin et al., 2005; Dedyukhin, 2010b; 2011c].

MATERIAL. Orenburg Prov.: 19 (FRc) Buzuluk distr., Buzulukskij Bor N.P., 12.V.2007 (K.A. Khristina); Bashkortostan: 4ex. (DSc) Sterlitamak, Shikhan Yuraktau, on Securegia varia, 29.V.2010 (S.V. Dedyukhin); Orenburg Prov.: 1 + (FRc) Buzuluk distr., Buzulukskij Bor N.P., 12.V.2007 (K.A. Khristina).

\section{Sitona lateralis Gyllenhal, 1834}

= Sitona ononidis Sharp, 1866.

RECORDS. VL: CHV KIR MAR* UDM* ULY TAT UR: BSH* CHL EKB KOM ORB* PER* [Csiki, 1901; Isaev, 1994; Mikhailov, 1999; Tshitshikov \& Legalov, 2008; Database of IEPA, 2009 (ononidis); Sapronov, 2010b; Dedyukhin \& Tselischeva, 2011]

MATERIAL. Mari El: $30^{\top} 2+(\mathrm{KUMN})$ Yoshkar-Ola, right bank of Malaya Kokshaga riv., $100 \mathrm{~m}$, N56 $39^{\prime} 12.1^{\prime \prime}$ E47 $56^{\prime}$ 07.7', meadow near Quercus-Abies forest, 13.VII.2006 (N.N Yunakov); 19 (ZIN) idem, 10.VIII.2009 (N.N. Yunakov); Udmurtia: 3ex. (DSc) Votkinsky distr., $7 \mathrm{~km} \mathrm{E}$ of Perevoznoye vill., Kama riv bank, 27.VI.2010, (S.V. Dedyukhin); Bashkortostan 1ex. (DSc) Sterlitamak, Shikhan Tratau, on Vicia cracca, 31.V.2010 (S.V. Dedyukhin); Perm' Reg.: 2ex. (DSc) Nytvensky distr., Sosnova vill., on Vicia cracca, 29.VI.2010 (S.V. Dedyukhin); Orenburg Prov.: 1ex. (RAc) 14 km NE from Orenburg, Grebeni vill., 14.VI.2006 (A.V. Rusakov); 1ex. (NVc) Belyaevka distr., Orenburgsky N.R.: Burtinskaya Steppe, 5.VII.2006 (V.A. Nemkov); 1ex. (NVc) idem, 6.VII.2008 (V.A. Nemkov); $2 \sigma^{7}$ (FRc) Sol'Iletsk distr., $5 \mathrm{~km}$ S from Pervomaiskoje vill., Shubaragash forest, 18-21.V.2007 (R.V. Filimonov).

\section{Sitona lepidus Gyllenhal, 1834}

= Sitona flavescens (Marsham, 1802) [HN].

RECORDS. VL: CHV KIR MAR* MRD TAT UDM ULY UR: CHL EKB PER [Lindeman, 1871 (flavescens); Lebedev, 1906; Jakovlev, 1910; Ryazantsev et al., 1966; Shernin, 1974; Roschinenko, 1981; Isaev, 1994; 1996; 2000; Dedyukhin et al., 2005; Tshitshikov \& Legalov, 2008; Database of IEPA, 2009; Sapronov, 2010b (flavescens); Dedyukhin, 2010d; Dedyukhin \& Tselischeva, 2011].

MATERIAL. Mari El: $30^{7}$ (ZIN) Yoshkar-Ola, right bank of Malaya Kokshaga riv., 100 m, N56 39' 12.1' 'E4756' 07.7' meadow near Quercus-Abies forest, 10.VIII.2009 (N.N. Yunakov).

\section{Sitona lineatus (Linnaeus, 1758)}

Curculio lineatus Linnaeus, 1758.

RECORDS. VL: CHV KIR MAR* MRD TAT UDM ULY UR: BSH* CHL EKB KOM ORB* PER [Lindeman, 1871; Lebedev, 1906; Jakovlev, 1910; Bei-Bienko, 1946; Ryazantsev et al., 1966; Roschinenko, 1972; Shernin, 1974; Isaev, 1994; 1996; Lagunov \& Novozhenov, 1996; Mikhailov, 1999; Dedyukhin et al., 2005; Tshitshikov \& Legalov, 2008; Database of IEPA, 2009; Dedyukhin, 2010d; Sapronov, 2010b; Dedyukhin \& Tselischeva, 2011].

MATERIAL. Mari El: $30^{7} 4$ ( $(\mathrm{ZIN})$ Medvedevo distr., Bolshaya Kokshaga N.R., nr Shaptunga vill., 150 m, N56 38' 31.92'' E4713' 45.41' meadow, sweeping, 29.VII.2006 (N.N. Yunakov); Bashkortostan: 1 ( (FRc) Mt. Iremel, 8.VII.1999 (E.V. Guskova); 1ex. (FRc) Burzyansky distr., Shulgan-Tash Nature Reserve, 3.VI.2000 (P.V. Rudoiskatel); Orenburg Prov.: 10 (FRc) Buzuluk distr., Buzulukskij Bor N.P., 12.VI.2007 (K.A. Khristina); $20^{7}$ (FRc) Sakmara distr., Mayorskoje vill., 24.V.2007 (R.V. Filimonov).

Sitona lineellus (Bonsdorff, 1785)

Curculio lineellus Bonsdorff, 1785.

RECORDS. UR: CHL EKB KOM ORB* PER [Bei-Bienko, 1946; Lagunov \& Novozhenov, 1996; Mikhailov, 1999; Tshitshikov \& Legalov, 2008; Database of IEPA, 2009].

MATERIAL. Orenburg Prov.: 1ex. (NVc) Belyaevka distr., Orenburgsky N.R.: Burtinskaya Steppe, 5.VII.2006 (V.A. Nemkov); 1ex. (NVc) Kuvandyk distr., Aituar vill., Orenburgsky N.R.: Aituarskaya Steppe, 5-10.VII.2002 (V.A. Nemkov).
Sitona longulus Gyllenhal, 1834

RECORDS. VL: CHV KIR* MRD TAT* UDM ULY UR: CHL EKB KOM ORB* [Koblova, 1967; Esyunin \& Kozminykh, 1992; Isaev, 1994; Mikhailov, 1999; Dedyukhin et al., 2005; Database of IEPA, 2009; Sapronov, 2010b].

MATERIAL. Tatarstan: 4ex. (DSc) Elabuga distr., Tanaika vill., meadow, on Medicago falcata, 21.VII.2010 (S.V. Dedyukhin); Kirov Prov.: 2ex. (DSc) Malmyzh distr., Gon'binka riv. bank, on Medicago falcata, 23.VI. 2010 (S.V. Dedyukhin). Orenburg Prov.: 4ex. (NVc) Belyaevka distr., Orenburgsky N.R.: Burtinskaya Steppe, fallow, 7.VII.2005 (V.A. Nemkov); 1 (FRc) Sol'-Iletsk distr., Novoiletsk vill., 26.V.2008 (R.V. Filimonov).

Sitona macularius (Marsham, 1802)

Curculio macularius Marsham, 1802.

= Sitona crinitus (Herbst, 1795) [HN]

RECORDS. VL: CHV KIR MAR* MRD SAM TAT UDM ULY UR: BSH* CHL EKB KOM ORB PER [Lindeman, 1871 (crinitus); Csiki, 1901 (crinitus); Lebedev, 1906; Jakovlev, 1910; Shernin, 1974; Bei-Bienko, 1946; Ryazantsev et al., 1966; Roschinenko, 1981; Isaev, 1994; 1996; 2000; Lagunov \& Novozhenov, 1996 (crinitus); Dedyukhin et al., 2005; Dedyukhin, 2011b; Dedyukhin \& Tselischeva, 2011].

MATERIAL. Mari El: $2 \sigma^{\top}$ (ZIN), Medvedevo distr., Bolshaya Kokshaga N.R., nr Shaptunga vill., $150 \mathrm{~m}$, N56 $38^{\prime} 31.92^{\prime \prime}$ E47 $13^{\prime}$ 45.41' meadow, sweeping, 29.VII.2006 (N.N. Yunakov); Bashkortostan: $2 \sigma^{\top} 1+$ (FRc) Mt. Iremel, 1000-1300m, 1-4.VI.2000 (R.V. Filimonov); Orenburg Prov.: $1 \sigma^{\top}$ (FRc) Sakmara distr., Mayorskoje vill., 24.V.2007 (R.V. Filimonov); $2 \sigma^{7}$ (FRc) Belyaevka distr., Orenburgsky N.R.: Burtinskaya Steppe, 26.V.2007 (V.A. Nemkov).

Sitona onerosus Faust, 1890

= Sitona uralensis L. Arnoldi, 1965.

= Sitona altaicus Khnzorian, 1970.

RECORDS. UR: ORB [Arnoldi et al., 1965].

Sitona puncticollis Stephens, 1831

RECORDS. VL: CHV KIR MAR* MRD UDM ULY UR: BSH* CHL EKB KOM PER [Jakovlev, 1910; Ryazantsev et al., 1966; Shernin, 1974; Isaev, 1994; Lagunov \& Novozhenov, 1996; Dedyukhin et al., 2005].

MATERIAL. Mari El: $20^{\top} 1$ ( $($ ZIN) Medvedevo distr., Bolshaya Kokshaga N.R., nr Shaptunga vill., 150 m, N56 $38^{\prime} 31.92^{\prime \prime}$ E47 $13^{\prime}$ 45.41" meadow, sweeping, 29.VII.2006 (N.N. Yunakov); Bashkortostan: $10^{7}$ (FRc) Mt. Iremel, mountain meadow, Malaise trap, 25.VIII.1992 (V.N. Olschwang).

\section{Sitona striatellus Gyllenhal, 1834}

= Curculio tibialis (Herbst, 1795) [HN] nec Sparrman, 1782. RECORDS. VL: CHV KIR MAR* MRD SAM TAT UDM ULY UR: BSH CHL EKB KOM ORB PER [Csiki, 1901 (tibialis); Lebedev, 1906; Jakovlev, 1910; Boitsova, 1931; Bei-Bienko, 1946; Ryazantsev et al., 1966; Shernin, 1974; Esyunin \& Kozminykh, 1992 (tibialis); Isaev, 1994; 1996; 2000; Mikhailov, 1999; Dedyukhin et al., 2005; Sapronov, 2010b (tibialis); Dedyukhin, 2011b].

MATERIAL. Mari El: $3 \sigma^{7}$ (ZIN) Yoshkar-Ola, right bank of Malaya Kokshaga riv., 100 m, N56 39' 12.1' 'E4756' 07.7' ' meadow near Quercus-Abies forest, 10.VIII.2009 (N.N. Yunakov); Bashkortostan: 14ex. (FRc) Burzyansky distr., Shulgan-Tash Nature Reserve, 3.VI.2000 (P.V. Rudoiskatel); Orenburg Prov.: 1 ex. (NVc) Tashla distr., Trudovoye vill., meadow, 6.VI.2006 (V.A. Nemkov); 1 ex. (NVc) Kuvandyk distr., Aituar vill., Orenburgsky N.R.: Aituarskaya Steppe, Shinbutak Ravine, 31.V.2007 (V.A. Nemkov).

Sitona sulcifrons (Thunberg, 1789)

Curculio sulcifrons Thunberg, 1789.

RECORDS. VL: CHV KIR MAR* MRD SAM TAT UDM ULY UR: BSH* CHL EKB KOM ORB* PER [Lebedev, 1906; Jakovlev, 1910; Kolosov, 1916; Boitsova, 1931; Bei-Bienko, 1946; Ryazantsev et al., 1966; Shernin, 1974; Roschinenko, 1981; Esyunin \& Kozminykh, 1992; Isaev, 1994; 1996; Lagunov \& Novozhenov, 
1996; Dedyukhin et al., 2005; Tshitshikov \& Legalov, 2008; Database of IEPA, 2009; Dedyukhin, 2010d; Sapronov, 2010b; Dedyukhin \& Tselischeva, 2011].

MATERIAL. Mari El: 10, 1 ( (ZIN) Medvedevo distr., Bolshaya Kokshaga N.R., nr Shaptunga vill., 150 m, N56 38' 31.92'' E47²13 45.41', meadow, sweeping, 29.VII.2006 (N.N. Yunakov); Bashkortostan: 1ex. (FRc) Burzyansky distr., Shulgan-Tash Nature Reserve, 4.VI.2000 (P.V. Rudoiskatel); Orenburg Prov.: 1ex. (RAc) Tyulgan distr., Tashla vill., meadow, 30.V.2006 (A.V. Rusakov); 3ex. (NVc) Gai distr., Guberla vill., steppe, 20.VII.2006 (V.A. Nemkov).

Sitona suturalis Stephens, 1831

RECORDS. VL: CHV KIR MAR* MRD SAM TAT UDM ULY UR: CHL EKB ORB* PER* [Csiki, 1901; Lebedev, 1925; Shernin, 1974; Isaev, 1994; 1996; Lagunov \& Novozhenov, 1996; Dedyukhin et al., 2005; Tshitshikov \& Legalov, 2008; Database of IEPA, 2009; Sapronov, 2010b; Dedyukhin \& Tselischeva, 2011].

MATERIAL. Mari El: $2 \sigma^{7}$ (ZIN) Yoshkar-Ola, right bank of Malaya Kokshaga riv., 100 m, N56³9' 12.1' E4756' 07.7', meadow near Quercus-Abies forest, 10.VIII.2009 (N.N. Yunakov); Orenburg Prov.: 1ex. (RAc) Buzuluk distr., Buzulukskij Bor N.P., 12.V.2007 (K.A. Khristina); 1ex. (NVc) Belyaevka distr., Orenburgsky N.R.: Burtinskaya Steppe, 5.VII.2006 (V.A. Nemkov); 1ex. (NVc) Gai distr., Guberla vill., steppe, 20.VII.2006 (V.A. Nemkov).

Sitona waterhousei Walton, 1846

RECORDS. VL: CHV UDM ULY [Egorov \& Isaev, 1994; Isaev, 1990; 1994; 1998; Dedyukhin, 2011a].

tribe ALOPHINI (2 genera, 2 species)

Graptus triguttatus (Fabricius, 1775)

Curculio triguttatus Fabricius, 1775.

RECORDS. VL: CHV KIR MRD SAM TAT UDM ULY [Lindeman, 1871; Csiki, 1901; Lebedev, 1906 (vau [MI, MS]); Roschinenko, 1972; Isaev, 1994; 1996; Dedyukhin, 2003; 2009; Dedyukhin et al., 2005].

Table 1. Sources and rate of knowledge of Entiminae in Volga and Ural Regions (per province). Таблица 1. Источники сведений и степень изученности Entiminae Поволжья и Урала (по регионам).

\begin{tabular}{|c|c|c|}
\hline Region/province & References & $\begin{array}{l}\text { Total number of } \\
\text { species recorded }\end{array}$ \\
\hline \multicolumn{3}{|l|}{ Volga } \\
\hline Chuvashia (CHV) & $\begin{array}{l}\text { Egorov \& Isaev, 1998; 2001; 2006; Isaev et al., 2004; Egorov, } \\
\text { 2004; Dmitrieva, 2005; Egorov \& Nikolaeva, 2005; Rutshin, } \\
2009\end{array}$ & $76(0)[\mathrm{MR} 2]$ \\
\hline Kirov (KIR) & $\begin{array}{l}\text { Jakovlev, 1910; Shernin, 1974; Yuferev, 2001a; 2001b; 2003; } \\
\text { 2004; Dedyukhin \& Tselischeva, 2011; Dedyukhin, 2011c }\end{array}$ & $52(1)[\mathrm{MR} 2]$ \\
\hline Mari El (MAR) & Matveev, 2007; Yunakov \& Korotyaev, 2007 & $25(23)$ \\
\hline Mordovia (MRD) & Lugovaya, 1970; Dmitrieva, 2005; Rutshin, 2009 & $67(1)$ \\
\hline Samara (SAM) & Dmitriev, 1935; Lona, 1938; Isaev, 1996; Isaev et al., 2004 & 63(3) [MR 1] \\
\hline Tatarstan (TAT) & $\begin{array}{l}\text { Lindeman, 1871; Csiki, 1901; Lebedev, 1906; 1912; Isaev et al., } \\
\text { 2004; Dedyukhin, 2001a; 2011c }\end{array}$ & $70(6)[\mathrm{MR} 1]$ \\
\hline Udmurtia (UDM) & $\begin{array}{l}\text { Roschinenko, 1972; 1981; Dedyukhin, 2003; 2006; 2009; } \\
\text { 2010b; 2010d; 2011a; 2011c; Dedyukhin et al., 2005; } \\
\text { Dedyukhin \& Tselischeva, } 2011\end{array}$ & $68(2)[\mathrm{MR} 6]$ \\
\hline Ulyanovsk (ULY) & $\begin{array}{l}\text { Korotyaev, 1984; Isaev, 1990; 1994; 2000; 2007; } \\
\text { Isaev et al., } 2004\end{array}$ & $83(0)$ \\
\hline \multicolumn{3}{|c|}{ 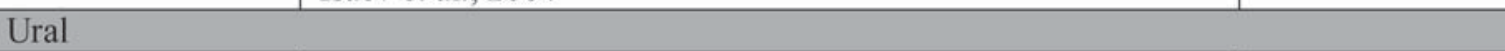 } \\
\hline $\begin{array}{l}\text { Bashkortostan } \\
\text { (BSH) }\end{array}$ & $\begin{array}{l}\text { Polozhentsev, 1947; Yunakov \& Korotyaev, 2007; Database of } \\
\text { IEPA, 2009; Dedyukhin, 2010c; 2011c }\end{array}$ & $48(26)[\mathrm{MR} 2]$ \\
\hline Chelyabinsk (CHL) & $\begin{array}{l}\text { Esyunin \& Kozminykh, 1992; Lagunov \& Novozhenov, 1996; } \\
\text { Mikhailov, 1999; Tshaschina, 2002; Tshitshikov \& Legalov, } \\
\text { 2008; Database of IEPA, 2009 }\end{array}$ & $68(10)[\mathrm{MR} 1]$ \\
\hline $\begin{array}{l}\text { Yekaterinburg } \\
\text { (EKB) }\end{array}$ & $\begin{array}{l}\text { Lindeman, 1871; Kolosov, 1916; Ermakov, 2003; } \\
\text { Bogacheva et al., 2009; Sapronov, 2008; 2010a; 2010b; } \\
\text { Database of IEPA, } 2009\end{array}$ & $40(1)[\mathrm{MR} 1]$ \\
\hline Komi (KOM) & $\begin{array}{l}\text { Sedykh, 1972; Lobanov, 1976; } \\
\text { Krylova, 1994; Medvedev et al., 2001; Database of IEPA, } 2009\end{array}$ & 28(3) [MR 1] \\
\hline Orenburg (ORB) & $\begin{array}{l}\text { Lindeman, 1871; Faust, 1881; 1888; Desbrochers, 1897; } \\
\text { Shiperovitsh 1939; Arnoldi, 1954; Koblova, 1967; Ismailova, } \\
\text { 1993; Nemkov \& Rusakov, 1995; Database of IEPA, 2009; } \\
\text { Dedyukhin, 2011b }\end{array}$ & $85(46)[\mathrm{MR} 4]$ \\
\hline Perm' (PER) & $\begin{array}{l}\text { Lindeman, 1871; Csiki, 1901; Chetyrkina, 1926; Baskina \& } \\
\text { Friedman, 1928; Boitsova, 1931; Bei-Bienko, 1946; } \\
\text { Ryazantsev et al., 1966; Dedyukhin, 2007; 2010a; 2011b }\end{array}$ & $49(13)$ \\
\hline
\end{tabular}

Number of new records is given in brackets; MR - number of misreported species.

Число новых указаний приведено в скобках; MR — число ошибочно указанных видов. 
Trichalophus korotyaevi Zherikhin et Nazarov, 1990

RECORDS. UR: BSH*.

MATERIAL. Bashkortostan: 1ex. (ZIN) Bolshoy Iremel Mt.R.,

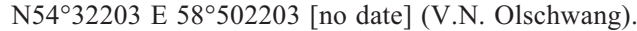

NOTE. This is a first record of Trichalophus LeConte, 1876 in the recent fauna of Europe.

\section{Species mistakenly recorded from the Volga and Ural regions}

Barynotus obscurus (Fabricius, 1775)

Curculio obscurus Fabricius, 1775.

RECORDS. VL: UDM [Roschinenko, 1972].

Otiorhynchus (Dorymerus) sulcatus (Fabricius, 1775)

RECORDS. VL: UDM [Roschinenko, 1972].

Otiorhynchus (Otiorhynchus) cornicinus Stierlin, 1861

= Curculio laevigatus Fabricius, 1792 [HN].

RECORDS. UR: BSH ORB [Faust, 1888].

Otiorhynchus minutus Stierlin, 1875

RECORDS. UR: ORB.

NOTE. Nomen dubium; for details see note to O. unctuosus.

Otiorhynchus transparens Stierlin, 1875

RECORDS. UR: ORB.

NOTE. Nomen dubium; for details see note to O. unctuosus.

Otiorhynchus (Prilisvanus) rugosus Hummel, 1827

RECORDS. VL: UDM [Roschinenko, 1972].

Phyllobius (Phyllobius) betulinus

(Bechstein et Scharfenberg 1805)

Curculio betulinus Bechstein et Scharfenberg 1805.

$=$ Curculio betulae (Fabricius, 1801) [HN], nec Linneaus, 1758

RECORDS.VL: CHV KIR SAM TAT UDM UR: BSH EKB

[Lebedev, 1906; Polozhentsev, 1947 (betulae); Shernin, 1974; Roschinenko, 1975; Isaev, 1994; 1996; Database of IEPA, 2009].

Phyllobius incanus (Gyllenhal,1834)

RECORDS.VL: UDM [Dedyukhin et al., 2005].

Rhinoscythropus vespertilio (Faust, 1884)

Phyllobius vespertilio Faust, 1884.

RECORDS.VL: KIR UDM UR: CHL [Esyunin \& Kozminykh, 1992; Roschinenko, 1972 (Phyllobius quercetorum)].

Polydrusus sericeus (Schaller, 1783)

Curculio sericeus Schaller, 1783.

RECORDS. VL: CHV UR: KOM [Sedykh, 1974; Egorov \& Isaev, 2001].

Polydrusus mus Desbrochers, 1897

RECORDS. UR: ORB.

NOTE. In original description the type locality is noted as "Caucase. Guberli". That is wrong because "Guberli" is name of river and village in Orenburg Province. The characters, mentioned by Desbrochers, suggest P. mus is conspecific with $P$. inustus.

\section{Strophosoma melanogrammum (Förster, 1771)}

Curculio melanogrammum Förster, 1771

RECORDS. MRD [Lugovaya, 1970].

NOTE. Likely this record from Mordovia was based on misidentified specimen(s) of S. capitatum (Deg.).

\section{Conclusions}

Volga and Ural regions comprises 113 Entiminae species. Phacephorus hirtellus Faust, 1885 and Polydrusus obliquatus Faust, 1884 are first records for Russian fauna. Trichalophus korotyaevi Zherikhin et Nazarov, 1990 is first record of the genus Trichalophus for recent fauna of Europe. Ural Mountains has no endemic species of Entiminae in contrast with other mountains of Eurasia, this ridge comprises very poor but heterogeneous Entiminae fauna. Orenburg Province has exclusive faunal composition where boreal species are neighboured with halophilous steppe species.

ACKNOWLEDGEMENTS. The authors thank V.O. Kozminykh (Perm'), V.A. Nemkov, A.V. Rusakov, K.A. Khristina, A.M. Shapovalov (Orenburg), V.E. Grigoryev (Sterlitamak), P.V. Rudoiskatel (Yekaterinburg), E.V. Guskova, and E.A. Chibiliov (Chelyabinsk) for supplying the material. Grateful appreciation is extended to B.A. Korotyaev (ZIN, St. Petersburg) for confirmation the identity of some species. The work of the first author was supported by research grant of the St. Petersburg Administration: PSP 1 070004 and by DFG (Deutsche Forschungsgemeinschaft) research grant: KL 1162/4-1.

\section{References}

Alonso-Zarazaga M.A. \& Lyal C.H.C. 1999. A World Catalogue of Families and Genera of Curculionoidea (Insecta: Coleoptera) (Excepting Scolytidae and Paltypodidae). London: Entomopraxis. 315 pp.

Aleynikova M.M. 1964. [Pochvennaya fauna Srednego Povolzh'ya ]. Moscow: Nauka. 173 pp [in Russian].

Arnoldi L.V. 1954. [Soil-dwelling insects larvae of Ural river area and Volga-Ural interfluve] // Trudy Zoologitsheskogo instituta AN SSSR. Vol.16. P.159-194 [in Russian].

Arnoldi L.V., Zaslavsky V.A. \& Ter-Minasian M.E. 1965. [82. fam. Curculionidae - weevils] // Opredelitel nasekomykh Evropeiskoy tshasti SSSR. Zhestkokrylye i Veerokrylye. Vol.2. Moscow-Leningrad: Nauka. P.485-621 [in Russian].

Arnoldi L.V., Ter-Minasian M.E. \& Solodovnikova V.S. 1974. [Fam. Curculionidae - weevils] // O.L. Kryzhanovski (ed.). Nasekomye i kleschi vrediteli sel'skokhozyaistvennykh kultur. Vol.2. Coleoptera. Leningrad: Nauka. P.218-293 [in Russian].

Arzanov Yu.G. \& Davidian G.E. 1995.[ A review of the weevils genus Nastus Schoenherr (Coleoptera, Curculionidae) of the fauna of the Crimea, European part of Russia and the Caucasus] // Entomologicheskoe obozrenie. Vol.74. No.3. P.622-639 [in Russian]

Baskina V.P. \& Friedman G.M. 1928. [Statistical study of animals in two ecological societies of Kama floodplain] // Izvestiya biologicheskogo nauchno-issledovatelskogo instituta i biologicheskoy stantsii pri Permskom universitete. Vol.1. No.2-3. P.183-295 [in Russian].

Bei-Bienko G.Ya. 1946. [Pests of the cultivated plants in the Molotov Prov.] Molotov: Molotov Univ. Publ. 132 pp. [in Russian].

Bogacheva I.A., Zamshina G.A. \& Sapronov V.V. 2009. [Curculionoidea beetles on deciduous trees and shrubs in Yekaterinburg] // Vestnik Chelyabinskogo Gosudarstvennogo Pedagogicheskogo Universiteta. No.9. P.310-321 [in Russian].

Boitsova M.K. 1931. [Fauna of lower layers of Pinetum cladinosum ]// Trudy Permskogo Biologitsheskogo Instituta. Vol.4. Iss.1-2. P.97-150 [in Russian].

Borovec R. 2009. Revision of the Palaearctic supraspecific taxa of the tribe Trachyphloeini (Coleoptera: Curculionidae: Entiminae) // Klapalekiana. Vol.45. P.1-97. 
Chetyrkina I.A. 1926. [Structure of the soil fauna of right bank of Kama River flood plain] // Izvestiya biologicheskogo nauchnoissledovatelskogo instituta i biologicheskoy stantsii pri Permskom universitete. Vol.5. No.2. P.61-89 [in Russian]

Csiki E. 1901 Coleopteren // Zichy E. Dritte asiatischen Forschungsreise des Grafen Eugen Zichy. Budapest-Leipzig. Bd.2. S.

Database of IEPA, 2009. Database of insect collection of Plant and Animal Ecology Institute, Ural Branch of Russian Academy of Sciences, Yekaterinburg // http://lib.ipae.uran.ru/museum [accessed 3.10.2011]

Davidian G.E. 1992. [Review of the weevil genus Pholicodes Schoenherr (Coleoptera, Curculionidae) of the fauna of Russia and neighboring countries] // Entomologicheskoe obozrenie. Vol.71 No.3. P.599-629 [in Russian].

Dedyukhin S.V. 2003. [Faunistic features and communities of Coleoptera in Udmurtia] // Vestnik Udmurtskogo Universiteta. Ser.Biologia. P.93-104 [in Russian].

Dedyukhin S.V. 2006. [Rare species of beetles (Coleoptera) of the steppe faunistic complex in Udmurtia] // Biodiversity and bioresources of Ural and adjacent territories. Proceedings of $3^{\text {rd }}$ International scientific conference. Orenburg: Print Service. P.175-177 [in Russian].

Dedyukhin S.V. 2007. [Interesting records of beetles (Hexapoda: Coleoptera) in scattered Kungur forest-steppe of Perm' Reg.] // Vestnik Udmurtskogo Universiteta. Ser. Biologia. No.10. P.7175 [in Russian].

Dedyukhin S.V. 2009. [To the knowledge of weevils (Coleoptera, Curculionoidea) of Netshkinsky National Park] // Vestnik Udmurtskogo Universiteta. Ser.6. Biologia. Nauki o Zemle. No.2. P.34-48 [in Russian].

Dedyukhin S.V. 2010a. [Phytophagous beetles (Coleoptera, Chrysomeloidea, Curculionoidea) of steppes faunistic complex in scattered Kungur forest-steppe] // Zoologicheskie issledovaniya v regionakh Rossii i na sopredel'nykh territoriyakh: Materialy mezhdunarodnoy nauchnoy konferentsii. Saransk: Progress. P.49-51 [in Russian].

Dedyukhin S.V. 2010b. [Results and prospects of the exploration of Curculionoidea and Chrysomeloidea beetles in plains of the eastern part of European Russia] // Entomological research in North Asia. Proceedings of VIII International conference of Siberian and Far East entomologists. 4-7.X.2010. Novosibirsk. P.69-71 [in Russian].

Dedyukhin S.V. 2010c. [Spring fauna of the phytophagous beetles (Coleoptera: Chrysomeloidea, Curculionoidea) on the limestone near Sterlitamak Mt.] // Proceedings of scientific conference "Study of natural and developed ecosystems of Ural and adjacent territories". 21-22.V.2010. Sterlitamak. P.63-68 [in Russian].

Dedyukhin S.V. 2010d. [Weevils (Coleoptera, Curculionoidea) in Botanical Garden of Udmurt University and arround: species, ecological occurance, and host plants] // Vestnik Udmurtskogo Universiteta. Ser.6. Biologia. Nauki o Zemle. Iss.4. P.42-55 [in Russian].

Dedyukhin S.V. 2011a. Checklist of beetles (Coleoptera) of the Udmurtia Republic. http://www.zin.ru/ANIMALIA/COLEOPTERA/RUS/udm list.htm [accessed 23.03.2011].

Dedyukhin S.V. 2011b. [Characteristics of the phytophagous beetles fauna in the north part of scattered Kungur forest-steppe] // Byulleten' Moskovskogo Obshchestva Ispytatelei Prirody. Otdel biol. Vol. No.2. P.20-28 [in Russian].

Dedyukhin S.V. 2011c. [Materials on the interesting findings of weevils (Coleoptera, Curculionoidea) in the east of the Russian plain] // Vestnik Udmurtskogo Universiteta. Ser.6. Biologia. Nauka o Zemle. 2011. No2. P.90-104 [in Russian].

Dedyukhin S.V., Nikitsky N.B. \& Semenov V.B. 2005. [Checklist of the beetles (Insecta, Coleoptera) of Udmurtia] // Eurasian entomological Journal. Vol.4. No.4. P.293-315 [in Russian].

Dedyukhin S.V.\& Tselischeva L.G. 2011. [Contribution to the fauna and ecology of Curculionoidea (Coleoptera) Nurgush Natural Reserve]// Nautshnye trudy Zapovednika Nurgush. Vol.1. P.1931 [in Russian].

Desbrochers J. 1897. Espèces inédites de Curculionides. VII (Suite) // Le Frelon. Vol.6. No.5 P.9-20.
Dmitriev G.V. 1935. [Contribution to the knowledge of entomofauna of Zhiguli Mountains] // Entomologicheskoe obozrenie. Vol.25. No.3-4. P.254-264 [in Russian].

Dmitrieva I.N. 2005. [Fauna and ecological characteristics of the weevils (Coleoptera, Curculionidae) in north of Volga upland forest-steppe]. Cheboksary. 180 pp. [in Russian].

Egorov L.V. 2004. [New and rare beetle species in the Chyuvashia (Insecta, Coleoptera). 2]//Vestnik Chuvashskogo Gosudarstvennogo Pedagogicheskogo Universiteta. No.4(42). P.162-175 [in Russian]

Egorov L.V. \& Isaev A.Yu. 1998. [To the knowledge of weevil fauna (Coleoptera: Apionidae, Curculionidae) of Chyuvashia Republic] // Entomologicheskie issledovaniya v Chuvashii. Materialy pervoi respublikanskoi entomologicheskoi konferentsii. Cheboksary. P.29-33 [in Russian].

Egorov L.V. \& Isaev A.Yu. 2001. [Addenda to the checklist of the weevils (Coleoptera: Apionidae, Curculionidae) of Chyuvashia Republic] // Vestnik Chuvashskogo Gosudarstvennogo Pedagogicheskogo Universiteta. No.1(20). P.59-67 [in Russian].

Egorov L.V. \& Isaev A.Yu. 2006. [On the structure of some weevil families (Coleoptera: Curculionoidea: Nemonychidae, Apionidae, Nanophyidae, Dryophthoridae, Erirhinidae, Curculionidae) of Chyuvashia] // Nauchnye trudy Gosudarstvennogo Prirodnogo Zapovednika "Prisurskii”". Cheboksary. Vol.13.P.1050 [in Russian].

Egorov L.V. \& Nikolaeva T.G. 2005. [Addenda to the fauna and ecology of the weevils (Insecta, Coleoptera, Curculionoidea) of Margaushsky distr., Chuvashia] // Materialy konferentsyi "Modernizatsiya obrazovaniya". 15.V.2005. Cheboksary. P.88105 [in Russian].

Ermakov A.I. 2003. [A review of the fauna and ecology of the weevils (Coleoptera, Curculionoidea) of Denezhkin Kamen' mountain massif(North-Urals)] // Euroasian entomological journal. Vol.1. No.2. P.229-234 [in Russian].

Esyunin S.L. \& Kozminykh V.O. 1992. [To the knowledge of the beetle fauna of "Troitsky" Protected Area] // Chlenistonogiye okhranayemykh terrioriy Chelyabinskoi Oblasti. Sverdlovsk: Uralskoe Otdelenie AN SSSR P.59-67 [in Russian].

Faust J. 1881. Beiträge zur Kenntniss der Käfer des Europäischen und Asiatischen Russlands mit Einschluss der Küsten des kaspischen Meeres. (3 Fortsetzung) // Horae Societatis Entomologicae Rossicae. T.16. No.3-4. S.285-333.

Faust J. 1888. Beiträge zur Kenntniss der Käfer des Europäischen und Asiatischen Russlands mit Einschluss der Küsten des kaspischen Meeres // Horae Societatis Entomologicae Rossicae. T.22. S.147-180.

Isaev A.Yu. 1990. [To the knowledge of weevil fauna (Coleoptera: Apionidae, Curculionidae) of Ulyanovsk Province] // Entomologicheskoe obozrenie. Vol.69. No.1. P.93-101 [in Russian].

Isaev A.Yu. 1994. [Ecological-faunistic review of weevils (Coleoptera: Apionidae, Rhynchophoridae, Curculionidae) from Ulyanovsk Province] // Priroda Ulyanovskoi oblasti. Pt.4. Ulyanovsk. 102 pp. [in Russian].

Isaev A.Yu. 1996. [Review of weevils (Coleoptera: Apionidae, Curculionidae) of Zhiguli Natural Reserve] // Samarskaya Luka. Vol.5 (1994). P.153-179 [in Russian].

Isaev A.Yu. 2000. [Additional data on the weevil fauna (Coleoptera, Curculionoidea: Coleoptera: Apionidae, Dryophthoridae, Curculionidae) of Ulyanovsk Province] // Nasekomye i paukoobraznye Ulyanovskoi oblasti. Priroda Ulyanovskoi oblasti. Vol.9. P.65-82 [in Russian].

Isaev A.Yu. 2007. [Key to the Coleoptera of Middle Volga. Pt.III. Polyphaga - Phytophaga]. Ulyanovsk: Vektor S. 256 pp. [in Russian].

Isaev A.Yu., Egorov L.V. \& Egorov K.A. 2004. [Beetles (Coleoptera) of the Middle Volga. Catalogue]. Ulyanovsk: Ulyanovsk State University Publ. 76 pp. [in Russian].

Ismailova M.Sh. 1993. [Review of the weevil genus Chlorophanus Germ. of Caucasus and remarks on the systematics of the tribe Tanymecini (Coleoptera, Curculionidae)]//Entomologicheskoe obozrenie. Vol.72. No.3. P.606-625 [in Russian].

Jakovlev A. 1910. Enumeratio coleopterorum a cl. L. Krulikovsky circum oppida Malmyzh (annis 1896-1899) et Urzhum (annis 
1899-1908) provinciae Vjatkensis (Rossiae mediae orientalis) collectorum // Trudy Russkogo Entomologicheskogo Obshchestva. Vol.39. P. 276-327.

Koblova T.A. 1967. [Fauna of Coleoptera of the south-eastern territories of Orenburg Province]. PhD thesis. Moscow. 228 pp. [in Russian].

Kolosov Yu.M. 1916. [Contribution to the knowledge of entomofauna of the Ural Mts. Pt.3. Addenda to the checklists of beetles (Coleoptera) of the Perm' hubernia] // Zapiski Ural'skogo obschestva lyubitelei yestestvoznaniya. Vol.35. No.11-12. P.239-244 [in Russian].

Korotyaev B.A. 1984. [Addenda to the fauna of weevil genus Phyllobius Germ. (Coleoptera, Curculionidae) of Mongolia and USSR] // Nasekomye Mongolii. Leningrad: Nauka Publ. Iss.9. P.356-365 [in Russian]

Korotyaev B.A. 1992. [New and little known species of weevils (Coleoptera: Curculionidae) from Russia and neighboring countries] // Entomologicheskoe obozrenie. Vol.71. No.4. P.807832 [in Russian].

Khrisanova M.A. \& Egorov L.V.2006. [A Review of Rhynchophorous Beetles (Coleoptera, Curculionoidea) of the Meshchera Lowland] // Entomologicheskoe obozrenie. Vol.85. No.3. P.580592 [in Russian].

Krylova L.P. 1994. [Invertebrates of Syktyvkar environs (Coleoptera)] // Animal ecology in natural and synanthrope habitats. Proceedings of the Komi research center UO RAN. Vol.136. P.60-79 [in Russian].

Lagunov A.V. \& Novozhenov Yu.I. 1996. [Beetle fauna of Ilmensky Natural Reserve]. Miass: IGZ UrO RAN Publ. 105 pp. [in Russian].

Lebedev A.G. 1906. [Contribution to the fauna of Kazan gubernia Pt.1] // Trudy Russkogo Entomologicheskogo Obshchestva.Vol.37. No.3-4. P.352-438 [in Russian]

Lebedev A.G. 1912. [Contribution to the fauna of Kazan gubernia. Pt.2] // Russkoe entomologicheskoe obozrenie. Vol.12. P.336348 [in Russian].

Lebedev A.G. 1925. [Contribution to the knowledge of beetle fauna of Tatarstan Republic] // Russkoe entomologicheskoe obozrenie. Vol.19. P.135-159 [in Russian]

Legalov A.A. 2010. Annotated checklist of species of superfamily Curculionoidea (Coleoptera) from Asian part of the Russia // Amurian zoological journal. Vol.2. No.2. P.93-132.

Lindeman K.E. 1871. [A reviev of geographical distirbution of beetles in Russian Impery] // Trudy Russkogo entomologicheskogo obshchestva. Vol.6. P.41-366 [in Russian].

Lobanov A.L. 1976. [New species of Coleoptera in fauna of Komi ASSR] // Abstracts of VII meeting "Biological problems of the North". Petrozavodsk. P.36-38 [in Russian]

Lona C. 1938. Curculionidae: Otiorrhynchinae III // S. Schenkling (ed.). Coleopterorum Catalogus auspiciis et auxilio W. Junk. Pars 162. Berlin. P.413-600.

Lugovaya L.A. 1970. [Towards the fauna of Middle Ural] // Uchenye zapiski MGPI imeni A.M. Gor'kogo. Vol.114. P.65-71 [in Russian].

Matveev V.A. 2007. [Changes of seasonal activities of dominating snout beetles (Curculionidae) due to forest fires in the Republic of Mari El] // Trudy zapovednika "Bolshaya Kokshaga". Yushkar-Ola: Mari State Univ. Publ. No.2. P.235-247 [in Russian]

Medvedev A.A., Lobanov A.L. \& Dolgin M.M. 2001. [New species of Coleoptera in fauna of NE European Russia] // Proceedings of the UrO RAN research center. No.166. P.15-19 [in Russian].

Mikhailov Yu.E. 1999. [Insects of museum-reserve "Arkaim". Beetles: species amount and population structure] // Natural systems of South Ural. Chelyabinsk: Chelyabinsk State University Publ. P.221-248 [in Russian].

Nemkov V.A. \& Rusakov A.V. 1995. [New data on the distribution of some insects species in South Ural steppes] // Zhivotnyi mir Yuzhnogo Urala i Severnogo Prikaspiya. Orenburg: Orenburg state pedadogic University publ. P.118-119 [in Russian].

Polozhentsev P.A. 1947. [Main insects species — pests of trees and shrubs in Bashkir Natural Reserve] // Trudy Bashkirskogo zapovednika. Iss.1. P.121-135 [in Russian].
Reitter E. 1915. Neue Übersicht der bekannten paläarktischen Arten der Coleoptera-Gattung Chloebius Schönh. // Wiener entomologische Zeitung. Bd.34. No.3-4. P.105-108.

Roschinenko V.I. 1972. [Arthropoda [part in chapter 'Fauna']. [checklist]] // A.I. Solov'ev (ed.). Priroda Udmurtii. Izhevsk: Udmurtia Publ. P.369-379. [in Russian].

Roschinenko V.I. 1981. [Ecological-zoogeographic characteristics of weevils and leav-rolling beetles of Udmurtia] // Fauna and ecology of animals of Udmurtia ASSR and adjacent territories. Izhevsk. P.99-106 [in Russian].

Roubal J. 1926. Sieben neuen Koleopteren aus der paläarktischen Region // Coleopterologische Centralblatt. Bd.1. P.244-249.

Ryazantsev A.V., Azheganova N.S. \& Matveenko G.G. 1966. [Pests of Trifolium pratense L. in Perm Province and data on their biology and ecology] // Proceedings of Perm agricultural institute. Vol.28(1965). P.183-193 [in Russian].

Rutshin A.B. 2009. Checklist of beetles (Coleoptera) of the Mordovia. http://www.zin.ru/ANIMALIA/COLEOPTERA/RUS/ colemord.htm [accessed 23.03.2011]

Sapronov V.V. 2008 [Addenda to weevil fauna of Middle Ural (Coleoptera, Curculionidae)] // Biosfera Zemli: proshloye, nastoyascheye $\mathrm{i}$ buduscheye. Proceedings of the young scientists conference, 21-25 April 2008. Yekaterinburg: Goschitsky Publ. P.269 [in Russian]

Sapronov V.V. 2010a. [Fauna of weevils (Coleoptera, Curculionidae) of boreal zone of the Ural] // Izvestiya Sankt-Peterburgskoi lesotekhnicheskoi akademii. No.192. P.215-219 [in Russian].

Sapronov V.V. 2010b. Weevil fauna of (Coleoptera, Curculionidae) of Yekaterinburg, feeding on grasses. http://ecotext.ru/93.html [access 23.03.2011]

Sedykh K.Th. 1974. [Fauna of Komi ASSR. Invertebrates]. Syktyvkar: Komi Publ. 192 pp [in Russian].

Seidlitz G. 1868. Die Otiorhynchiden sensu str. nach den morphologischen Verwandtschaft-Verhältnissen ihres Hautsceletts vergleichend dargestellt // Berliner Entomologische Zeitschrift Bd.12. Beiheft. S.1-153.

Shernin A.I. 1974. [Order Coleoptera. Beetles] // Fauna of Kirov Province. Vol.2. Kirov: VGPU Publ. P.111-227 [in Russian].

Shiperovitsh V.Ya. 1939. [Soil and arboreal fauna of different forest types in the Buzuluksky Bor Reserve]// Zoologicheskyi zhurnal. Vol.18. No2. P.196-211 [in Russian].

Stierlin G. 1875. Vierter Nachtrag zur Revision der europäischen Otiorhynchus-Arten // Berliner Entomologische Zeitschrift. Vol.19. P.337-354.

Tshaschina O.E. 2002. [Contribution to the beetle fauna (Insecta: Coleoptera) of Ilmenskyi Reserve] // Izvestia Chelyabinskogo nauchnogo centra. Vol.2. No.15. P.73-78 [in Russian].

Tshitshikov B.M. \& Legalov A.A. 2008. [Checklist of weevils (Coleoptera, Curculionoidea) agricultural lands of South Ural] // Izvestia Chelyabinskogo nauchnogo centra. Vol.2. No.40. P.26-30 [in Russian].

Wells S.M. \& Chatfield J.E. 1992. Threatened non-marine mollusks of Europe. Nature and environment. 64. Strasbourg: Council of Europe Publ. 170 pp.

Yuferev G.I. 2001a. [Order Coleoptera. Beetles] // Fauna of Kirov Province. Addenda. Vol.5. Kirov: VGPU Publ. P.120-180 [in Russian].

Yuferev G.I. 2001b. Addenda to the checklist of Coleoptera of the Kirov Province.http:/www.zin.ru/ANIMALIA/COLEOPTERA/ RUS/vyat_add.htm [accessed 23.03.2011]

Yuferev G.I. 2003. Second addendato the checklist of Coleoptera of the Kirov Province. http://www.zin.ru/ANIMALIA/COLEOPTERA/RUS/vyatadd2.htm [accessed 23.03.2011]

Yuferev G.I. 2004. [Insect fauna of Kirov Province. New data]. Kirov: Triada Plus. 24 pp. [in Russian]

Yunakov N.N. 2005. [A revision of the weevil genus Amicromias Rtt. (Coleoptera: Curculionidae: Entiminae)] // Entomologicheskoe obozrenie. Vol.85. No.1. P.156-168 [in Russian].

Yunakov N.N. \& Korotyaev B.A. 2007. [Review of the subgenus Metaphyllobius Smirnov (Coleoptera, Curculionidae, Entiminae) of East Europe and Siberia] // Entomologicheskoe obozrenie. Vol.86. No.3. P.687-703 [in Russian] 